OPEN ACCESS

Edited by:

Dimitris G. Hatzinikolaou,

National and Kapodistrian University

of Athens, Greece

Reviewed by:

Brett I. Pletschke,

Rhodes University, South Africa

Neeta Agarwal,

Indian Veterinary Research Institute

(IVRI), India

*Correspondence: Xiangshu Piao

piaoxsh@cau.edu.cn

Specialty section:

This article was submitted to

Systems Microbiology,

a section of the journal

Frontiers in Microbiology

Received: 07 May 2021

Accepted: 11 June 2021

Published: 15 July 2021

Citation:

Wang J, Liu S, Ma J and Piao X (2021) Changes in Growth Performance and lleal Microbiota

Composition by Xylanase Supplementation in Broilers Fed Wheat-Based Diets.

Front. Microbiol. 12:706396. doi: 10.3389/fmicb.2021.706396

\section{Changes in Growth Performance and Ileal Microbiota Composition by Xylanase Supplementation in Broilers Fed Wheat-Based Diets}

\author{
Jian Wang, Sujie Liu, Jiayu Ma and Xiangshu Piao* \\ State Key Laboratory of Animal Nutrition, College of Animal Science and Technology, China Agricultural University, Beijing, \\ China
}

Xylanase exerts key roles in improving growth performance and intestinal health of broilers fed wheat-based diets. However, knowledge is limited regarding effects of xylanase supplementation on ileal microbiota in broilers. A total of 128 one-day-old broilers (initial BW $48.03 \pm 0.33 \mathrm{~g}$ ) were selected to investigate effects of xylanase (AT-xynA) on growth performance, ileal morphology, microbiota composition, immune response, antioxidant capacity, and endocrine peptide levels in broilers. Broilers were randomly allotted into two dietary treatments $(n=8)$, namely, a wheat-soybean basal diet and a basal diet with 4,000 U/kg AT-xynA (XY). On days 7, 14, 21, and 42, broilers were weighted and ileal tissues were sampled. lleal digesta samples were collected for analyzing microbiota composition on days 21 and 42 . The results showed that AT-xynA could improve average daily weight gain and average daily feed intake, and there were interactions between diet and age of broilers $(p<0.05)$. On days 21 and 42 , xylanase supplementation decreased ileal microbiota $\alpha$-diversity, and the relative abundance of potentially pathogenic microbiota, such as phylum Proteobacteria, family Moraxellaceae and Staphylococcaceae, genus Staphylococcus, Pseudomonas, Streptococcus, and Enterococcus, increased the abundance of Lactobacillus ( $p<0.05)$. Moreover, the reduction in acetate concentration and abundance of short-chain fatty acid-producing bacteria was also observed in broilers from $X Y$ group $(p<0.05)$. AT-xynA increased ileal villus height, glucagon-like peptide-1, and insulin-like growth factor- 1 concentrations and decreased interleukin-1 $\beta$, interleukin-6, tumor necrosis factor- $\alpha$, and malondialdehyde content in broilers, and these positive effects on intestinal health were greater in young broilers. In conclusion, xylanase supplementation to wheat-based diets could improve ileal intestinal morphology and immune function, and alleviate excess fermentation of bacteria, which may be related to changes of intestinal microbiota. In addition, the positive effects of xylanase on intestinal health were more pronounced in young broilers, thus contributing to subsequent improvement in growth performance of broilers.

Keywords: xylanase, wheat, broiler, ileal, microbiota 


\section{INTRODUCTION}

Wheat can be included in poultry diets as a major energy source, especially in European broilers diets (Ball et al., 2013). However, the non-starch polysaccharides (NSP) present in wheat cell walls showed anti-nutritional effects, which might negatively affect broilers performance (Smeets et al., 2018). The increase of digesta viscosity in the small intestine due to soluble NSPs in wheat is a major reason for anti-nutritional effects, and the increase will reduce diffusion rate of substrates and digestive enzymes, inhibit effective interaction between them in the intestine, thus reduce digestion and absorption of nutrients, and result in poor performance (Choct et al., 1996; Bedford and Schulze, 1998). Moreover, negative effects of soluble NSPs on performance might be indirectly associated with microbiota composition in the small intestine, soluble NSPs lead to a slow digesta flow, decrease small intestine oxygen tension, and thereby promote the growth of anaerobic microorganisms, and the changes of microbiota composition could negatively affect physiological function of the intestine (Choct et al., 1996; Raza et al., 2019). Therefore, the application of NSP degrading enzymes in wheat-based diets is necessary to improve growth performance and intestinal health of broilers.

Arabinoxylans are the major NSPs in wheat, the content of arabinoxylan ranges from 4.1 to $9.0 \%$, and thus, endo- $\beta$ 1,4 -xylanases are commonly supplemented in broilers wheatbased diets for degradation of arabinoxylans (Rosicka-Kaczmarek et al., 2016; Cardoso et al., 2018). It is generally believed that the mechanism of NSP-degrading enzymes in broilers includes reducing digesta viscosity, releasing encapsulated nutrients, and providing fermentable NSP hydrolysis products (Masey et al., 2014). At the present, positive effects of xylanase on growth performance of broilers have been well accepted; however, the effect of xylanase on intestinal health is still an active field of research (Choct et al., 1999; Cardoso et al., 2018). The xylanase is generally considered to work through two stages, namely, an ileal stage and a cecal stage (Engberg et al., 2004). Most studies focused on effects of xylanase on cecal microbiota; the knowledge regarding ileal microbiota was limited (McCafferty et al., 2019a,b; Petry et al., 2021). In fact, NSP-degrading enzyme improves small intestine digestion as a consequence decreases undigested substrates in the small intestine, which might also result in the reduction of fermentative microorganisms and potentially pathogenic microorganisms in the ileum (Danicke et al., 1999; Raza et al., 2019). At the present, effects of xylanase on ileal microbiota composition were just analyzed by conventional molecular ecology techniques; thus, changes of microbiota composition still need to be further explored (Hübener et al., 2002; Engberg et al., 2004). 16S rRNA gene sequencing has been proved to be a powerful tool for the assessment of diversity and composition of microbiota due to its informative and predictive potential. Moreover, there are interactions between ileal microbiota and immune responses, and the higher pathogen loads in the small intestine could also contribute to oxidative stress and inflammatory responses (Tiwari et al., 2018). Therefore, we performed an experiment to determine effects of xylanase on growth performance, intestinal morphology, immune response, antioxidant capacity, barrier function, and endocrine peptide levels of broilers. Subsequently, ileal microbiota was analyzed through high-throughput sequencing of the 16S rRNA gene, and correlations between microbiota composition with intestinal morphology and immunity function were evaluated.

\section{MATERIALS AND METHODS}

The Laboratory Animal Welfare and Animal Experimental Ethical Inspection Committee of China Agricultural University (Beijing, China) approved all the experimental protocols in this study (AW70601202-1-1).

\section{Preparation of Xylanases}

The xylanase (AT-xynA) in this study was provided by the Ministry of Agriculture Feed Industry Center Lab in China Agricultural University (Beijing, China). The xylanase (AT-xynA) was prepared as previously described (Wang et al., 2020). One unit of activity is defined as the amount of xylanase that releases $1 \mu \mathrm{mol}$ of xylose per min at the optimal conditions (pH 3.0 and $50^{\circ} \mathrm{C}$ ).

\section{Experiment Animals, Management, Design, Diets, and Processing Procedure}

The 128 one-day-old broilers (Arbor Acres Poultry Breeding Company, Beijing, China) with initial body weight $48.03 \pm 0.33 \mathrm{~g}$ were randomly divided into two treatments, and each treatment consisted of eight replicates with eight broilers in each replicate. The two dietary treatments consisted of a wheat-soybean basal diet (CON) and a CON with 4,000 U/kg AT-xynA (XY). Experimental diets were supplied throughout two phases: a starter phase (days 0-21) and a grower-finisher phase (days 21-42). The ingredients of experimental diets were exhibited (Table 1), and all essential nutrients of the basal diet satisfied nutrient requirements of broilers following National Research Council (1994) recommendations. The management of broilers in this experiment followed the guidelines of raising AA broilers described by Mahfuz et al. (2019).

\section{Growth Performance, Sample Collection, and Intestinal Morphology Analysis}

Body weight and feed intake were recorded on days $0,7,14$, 21 , and 42 to calculate average daily weight gain (ADG), average daily feed intake (ADFI), and feed conversion ratio (FCR) of broilers. On days 7, 14, 21, and 42, one broiler from each replicate was slaughtered for intestinal sample collection, and middle parts of ileum were collected and then immediately placed in $10 \%$ buffered formalin. The formalin-fixed tissues were embedded in paraffin and cut into about $5 \mathrm{~mm}$ slices. Then, samples were dyed with hematoxylin and eosin (HE) for histological examination, and six well-oriented crypt and villi from each slice were selected for determination of the villi height $(\mathrm{VH})$ and crypt depth (CD) through a light microscope. Ileal tissue samples of slaughtered broilers from 
TABLE 1 | Composition and nutrient levels of basal diets (\%, as-fed basis).

\begin{tabular}{lrc}
\hline Item & days $\mathbf{0 - 2 1}$ & days 21-42 \\
\hline Ingredients & & \\
Corn & 33.16 & 37.45 \\
Wheat & 33.00 & 31.91 \\
Soybean meal, 43\% & 23.05 & 19.22 \\
Fish meal, 64.6\% & 3.62 & 3.74 \\
Soybean oil & 3.60 & 4.50 \\
Dicalcium phosphate & 1.17 & 1.09 \\
Limestone & 1.24 & 1.05 \\
Salt & 0.30 & 0.30 \\
L-lysine HCl, 78\% & 0.12 & 0.11 \\
DL-methionine, 98\% & 0.16 & 0.05 \\
L-threonine, 98\% & 0.08 & 0.08 \\
Vitamin-mineral premix & 0.50 \\
Total & 0.50 & 100.00 \\
Nutrient content & 100.00 & \\
Metabolizable energy, & & 3,150 \\
kcal/kg & 3,050 & 18.95 \\
Crude protein & & 0.90 \\
Calcium & 20.53 & 0.65 \\
Total phosphorous & 1.00 & 1.00 \\
Lysine & 0.68 & 0.38 \\
Methionine & 1.10 & \\
\hline
\end{tabular}

aVitamin A, 11,000 IU; vitamin D, 3,025 IU; vitamin E, $22 \mathrm{mg}$; vitamin K3, $2.2 \mathrm{mg}$; thiamine, $1.65 \mathrm{mg}$; riboflavin, $6.6 \mathrm{mg}$; pyridoxine, $3.3 \mathrm{mg}$; cobalamin, $17.6 \mu \mathrm{g}$; nicotinic acid, $22 \mathrm{mg}$; pantothenic acid, $13.2 \mathrm{mg}$; folic acid, $0.33 \mathrm{mg}$; biotin, $88 \mu \mathrm{g}$; choline chloride, $500 \mathrm{mg}$; iron, 48 mg; zinc, 96.6 mg; manganese, 101.76 mg; copper, 10 mg; selenium, 0.05 mg; iodine, $0.96 \mathrm{mg}$; and cobalt, $0.3 \mathrm{mg}$.

${ }^{b}$ Values are calculated according to NRC.

each treatment were also collected on days $7,14,21$, and 42 and then maintained at $-80^{\circ} \mathrm{C}$. On days 21 and $\mathrm{d} 42$, broilers ileal digesta from each treatment were sampled to analyze composition and metabolites of the intestinal microbiota.

\section{Microbiota Profiling}

The extraction for total bacterial genomic DNA of ileal digesta samples was performed using the EZNA Stool DNA kit (Omega Bio-Tek, Norcross, GA, United States) following instructions of manufactures. The PCR conditions for amplification of the $\mathrm{V} 3-\mathrm{V} 4$ region of the bacterial $16 \mathrm{~S}$ rRNA gene were as follows: $3 \mathrm{~min}$ at $95^{\circ} \mathrm{C}, 27$ cycles of $95^{\circ} \mathrm{C}$ for $30 \mathrm{~s}, 55^{\circ} \mathrm{C}$ for $30 \mathrm{~s}$, $72^{\circ} \mathrm{C}$ for $45 \mathrm{~s}$, and $10 \mathrm{~min}$ at $72^{\circ} \mathrm{C}$. The $16 \mathrm{~S}$ rRNA gene was amplified with primers 338F (5'barcodeACTCCTAC GGG AGGCAGCAG3') and 806R (5'GGACTACHVGGGTWTC TAAT3'). The $2 \%$ agarose gel-electrophoresis was used for the detection of purity of PCR products, and the products were purified with the AxyPrep DNA Gel Extraction kit (Axygen Biosciences, Union City, CA, United States). Purified amplicons were quantified by QuantiFluor (Promega, Madison, WI) and then paired-end sequenced $(2 \times 250)$ on the Illumina MiSeq platform (Illumina, San Diego, CA, United States). Raw fastq files were de-multiplexed and quality-filtered with QIIME (version 1.70). Operational taxonomic units (OTUs) were clustered using a 97\% similarity cut-off with UPARSE (version 7.1), and chimeric sequences were removed using UCHIME. Representative sequence of OTUs was obtained based on Ribosomal Database Project classifier. The sequence data were uploaded to the NCBI Sequence Read Archive database (accession number: PRJNA725811).

\section{Microbial Metabolites}

As reported by previous study, short chain fatty acid (SCFA) concentrations in ileal digesta were detected by using an ion chromatographic method (Liu et al., 2017). About $0.5 \mathrm{~g}$ of ileal digesta sample was mixed with $8 \mathrm{ml}$ of distilled water, and after $30 \mathrm{~min}$ of ultrasonic treatment, the mixture was centrifuged at $8,000 \times \mathrm{g}$ for $10 \mathrm{~min}$ to achieve the supernatant. We filtered the diluted supernatant using a $0.22 \mu \mathrm{m}$ filter and finally injected the supernatant into a high-performance ion chromatograph (ICS 3000 Dionex, United States). SCFAs were separated by an AG11 guard column and an AS11 analytical column under the gradient condition (0-5 $\mathrm{min}, 0.8-1.5 \mathrm{~mm}$; 5-10 $\mathrm{min}, 1.5-2.5 \mathrm{~mm}$; and $10-15 \mathrm{~min}, 2.5 \mathrm{~mm}$ ); the flow rate was $1.0 \mathrm{ml} / \mathrm{min}$. The gradient was carried out with potassium hydroxide.

\section{Tight Junction Gene Expression}

Total RNA of ileal tissues was isolated by using mirVana Kit (Ambion) following the manufacturer's directions. Then, RNA yield was measured by a NanoDrop 2000 spectrophotometer (Thermo Fisher Scientific, Waltham, MA, United States), and RNA quality was assessed by $1 \%$ agarose gels electrophoresis. PrimeScript RT reagent kit (TaKaRa, China) was used to complete the reverse transcription following the manufacture's recommendations. Real-time PCR was performed with LightCycler ${ }^{\circledR} 480$ II Real-time PCR Instrument (Roche, Swiss), and the mixture consisted of $1 \mu \mathrm{l}$ of cDNA, $5 \mu \mathrm{l}$ of $2 \times$ PerfectStart $^{\mathrm{TM}}$ Green qPCR SuperMix, $0.2 \mu$ l of forward primer, $0.2 \mu \mathrm{l}$ of reverse primer, and $3.6 \mu \mathrm{l}$ of nuclease-free water. The reaction conditions were $30 \mathrm{~s}$ at $94^{\circ} \mathrm{C}, 45$ cycles of $5 \mathrm{~s}$ at $94^{\circ} \mathrm{C}$, and $30 \mathrm{~s}$ at $60^{\circ} \mathrm{C}$. After the $\mathrm{qPCR}$ run, the expected PCR product was recognized by analyzing melting curve. The primers for genes associated with intestinal barrier function including zonula occludens-1 (ZO-1; forward primer: 5'AGATGGACAGCATCAACG3'; reverse primer: 5'CTGCCAC ATCCTG GTATT3'), occludin (forward primer: 5'TGGT ACTGACCAACGTAGTTC3'; reverse primer: 5'AGGAGTGAC ATCTAATAAAGCG3'), and housekeeping genes [ $\beta$-actin and glyceraldehyde phosphate dehydrogenase (GAPDH); forward primer: 5'GAAGGCTGGGGCTCATCTG3'; reverse primer: 5'CAGTTGGTGGTGCACGA TG3'] were synthesized by TsingKe Biotech (Beijing, China). The relative expression of ZO-1 and occludin gene was analyzed by the $2^{-\Delta \Delta \mathrm{Ct}}$ method and normalized to reference gene (GAPDH) (Livak and Schmittgen, 2001).

\section{Intestinal Antioxidant and Immune Function}

The malondialdehyde (MDA) concentration and the activity of superoxide dismutase (SOD) in the ileum were measured using commercial assay kits (Nanjing Jiancheng Bioengineering Institute, Nanjing, China) following the manufacturer's instructions. The levels of interleukin-1 $\beta$ (IL-1 $\beta$ ), interleukin-6 (IL-6), interleukin-10 (IL-10), tumor necrosis factor- $\alpha$ (TNF- $\alpha$ ), and interferon- $\gamma($ IFN- $\gamma$ ) in the ileum were determined by 
enzyme-linked immunosorbent assay (ELISA) kits (Nanjing Jiancheng Bioengineering Institute, Nanjing, China).

\section{Endocrine Peptides}

The determination of insulin-like growth factor 1 (IGF-1), epidermal growth factor (EGF), glucagon-like peptide-1 (GLP-1), and glucagon-like peptide-2 (GLP-2) levels in the ileum was detected with the ELISA kits (Laibo Tairui Technology Development Co., Ltd., Beijing, China) according to manufacturer's instructions.

\section{Statistical Analysis}

The data analysis was performed by using the procedure of SAS (version 9.2, 2008), and results were expressed as means \pm standard error of the mean (SEM). When evaluating growth performance, the replicate was considered as an experimental unit. For other parameters (intestinal morphology, tight junction gene expression, antioxidant activities, inflammation function, endocrine peptide levels, and SCFA concentrations), the individual slaughtered broiler was treated as an experimental unit. The student's test procedure was performed to compare two groups: $p<0.05$ was found to be statistically significant, whereas $0.05 \leq p \leq 0.10$ was regarded as a significance of trend. The $\alpha$-diversity indices, including Shannon diversity index (Shannon), ACE estimator (Ace), Chao 1 estimator (Chao), observed richness (Sobs), Good's coverage (Coverage), and phylogenetic diversity (PD), were calculated by using QIIME. The procedure of the linear discriminant analysis (LDA) effect size linear discriminant analysis (LEfSe) algorithm was used to find differences in the relative abundance of bacteria between two treatments with LDA score of bacteria exceeding 2.0. We used R package of "Hmisc" to calculate the Spearman's correlation coefficient. Functions of bacterial community from ileal digesta samples were predicted by PICRUSt analysis.

\section{RESULTS}

\section{Growth Performance}

As given in Table 2, ADG, ADFI, and FCR of broilers were significantly affected by age $(p<0.01)$. The addition of AT-xynA could significantly improve ADG $(p<0.01)$ and ADFI $(p=0.030)$ of broilers, and tended to improve FCR $(p=0.056)$. Broilers from XY group showed higher ADG than those from CON group from days $14-21$ and days $21-42(p<0.05)$. Compared with broilers fed with no AT-xynA, broilers fed diets containing AT-xynA also exhibited higher ADFI during days 21-42 and lower FCR during the third week $(p<0.05)$. For ADG $(p<0.01)$ and $\operatorname{ADFI}(p=0.014)$, there were significant interactions between diet and age of broilers.

\section{Ileal Morphology}

The light micrographs of ileal morphology are displayed in Figure 1, and data about the ileal morphology are given in Table 3. Compared with CON group, $\mathrm{VH}$ of ileum was greater in broilers from XY group on day $7(p=0.015)$ and day 21 $(p=0.010)$, and VH of ileum also showed a trend to increase in broilers from XY group $(p=0.097)$ on day 14 . However, no differences were shown in $\mathrm{CD}$ and $\mathrm{VH} / \mathrm{CD}$ values between two treatments.

\section{Microbial Diversity and Community in Ileal Digesta}

As shown in Figure 2, the $\alpha$-diversity analysis, Shannon $(p=0.011)$, Ace $(p=0.044)$, and Chao $(p=0.009)$ indices were significantly lower in the ileum of broilers fed with AT-xynA, Sobs $(p=0.089)$ and $\mathrm{PD}(p=0.078)$ indices showed trends to decrease in broilers from XY group on day 21. On day 42, broilers from $\mathrm{XY}$ group also showed lower Sobs $(p=0.041)$ and $\mathrm{PD}(p=0.033)$ indices compared with broilers from CON group.

We assessed the ileal microbiota at various taxonomic levels to evaluate differences in the microbiota composition induced by addition of AT-xynA. At the phylum level, the dominant bacterial group in CON group was Firmicutes, Actinobacteriota, Proteobacteria, Bacteroidota, Cyanobacteria, Deinococcota, Verrucomicrobiora, and Acidobacteria, and Firmicutes, Actinobacteriota, Proteobacteria, Bacteroidota, Cyanobacteria, and Verrucomicrobiora were the major phyla in XY group on day 21. In addition, on day 42 , ileal microbiota was dominated by Firmicutes, Actinobacteriota, Proteobacteria, Bacteroidota, Cyanobacteria, and Verrucomicrobiora in CON group, and the major phyla in ileal microbiota of broilers from XY group were Firmicutes, Actinobacteriota, Proteobacteria, and Bacteroidota. As shown in Figure 3, the abundance of Proteobacteria significantly decreased in XY group compared with that in CON on day $21(p=0.042)$ and day $42(p=0.033)$. In contrast, the abundance of Firmicutes in XY group was higher than broilers fed diets un-supplemented AT-xynA on day 21 and day 42 but no significant differences were observed (day 21, CON: $88.82 \%$ vs. XY: $99.25 \%$; day 42 , CON: $98.89 \%$ vs. XY: $99.84 \%$ ).

At the family level, the most dominant families (> 1\%) in the ileum of broilers from CON group included Lactobacillaceae (71.83\%), Micrococcaceae (6.66\%), Clostridiaceae (5.23\%), Lachnospiraceae (3.05\%), Streptococcaceae (3.98\%), Corynebacteriaceae (1.18\%), and Staphylococcaceae (1.04\%), whereas Lactobacillaceae $(94.28 \%)$ and Lachnospiraceae (2.24\%) were the most dominant families $(>1 \%)$ in XY group on day 21 . On day 42, ileal microbiota was both dominated $(>1 \%)$ by Lactobacillaceae (CON: $94.23 \%$ vs. XY: $99.31 \%$ ) in $\mathrm{CON}$ and $\mathrm{XY}$ groups. As shown in Figure 4, on day 21, the abundance of Lactobacillaceae $(p=0.019)$ was higher in ileal microbiota of broilers from $\mathrm{XY}$ group compared with those from CON group. In contrast, the abundance of Microbacteriaceae $(p=0.012)$, Exiguobacteraceae $(p=0.043)$, Rhizobiaceae $(p=0.040)$, Beijerinckiaceae $(p=0.045)$, and Propionibacteriaceae $(p=0.016)$ was higher in broilers from CON group on day 21. On day 42 , the abundance of Lactobacillaceae also increased in ileal microbiota of broilers from $\mathrm{XY}$ group $(p=0.005)$, whereas the abundance of Bacillaceae $(p<0.01)$, 
TABLE 2 | Effects of AT-xynA on growth performance of broilers.

\begin{tabular}{|c|c|c|c|c|c|c|c|c|c|c|c|c|}
\hline \multirow{2}{*}{ Items } & \multicolumn{2}{|c|}{ days $0-7$} & \multicolumn{2}{|c|}{ days $7-14$} & \multicolumn{2}{|c|}{ days $14-21$} & \multicolumn{2}{|c|}{ days $21-42$} & \multirow{2}{*}{ SEM } & \multicolumn{3}{|c|}{ Value of $p$} \\
\hline & CON & $\mathbf{X Y}$ & CON & $\mathbf{X Y}$ & CON & $\mathbf{X Y}$ & CON & $\mathbf{X Y}$ & & Diet & Age & Diet*Age \\
\hline Average daily feed intake, $\mathrm{g}$ & $15.00^{\circ}$ & $15.18^{e}$ & $22.28^{d}$ & $23.30^{d}$ & $56.18^{\mathrm{c}}$ & $56.38^{\circ}$ & $89.61^{b}$ & $98.89^{a}$ & 1.57 & 0.030 & $<0.01$ & 0.014 \\
\hline Feed conversion ratio & $1.06^{d}$ & $1.04^{d}$ & $1.09^{d}$ & $1.08^{d}$ & $1.52^{\mathrm{b}}$ & $1.40^{\circ}$ & $1.65^{\mathrm{a}}$ & $1.74^{\mathrm{a}}$ & 0.04 & 0.056 & $<0.01$ & 0.482 \\
\hline
\end{tabular}

${ }^{1}$ CON $(n=8)$ is AT-xynA un-supplemented group; $X Y(n=8)$ is 4,000 U/kg AT-xynA supplemented group. a-t Means with a row with no common superscript differ significantly.

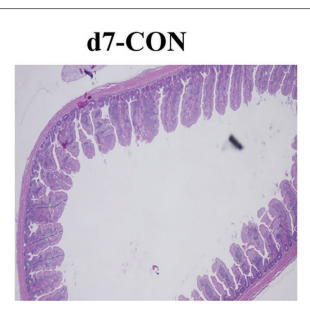

d7-XY

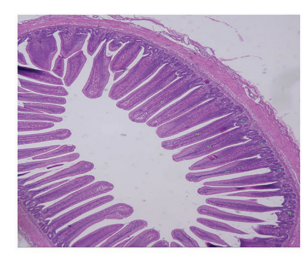

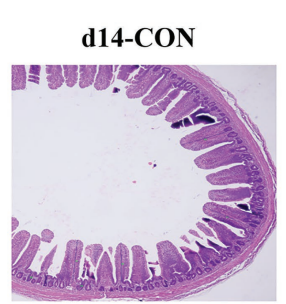

d14-XY

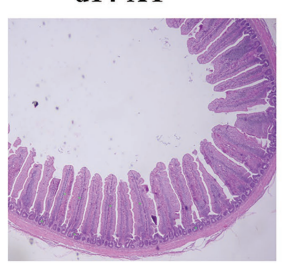

d21-CON

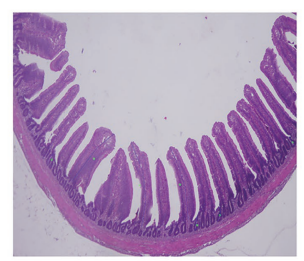

d21-XY

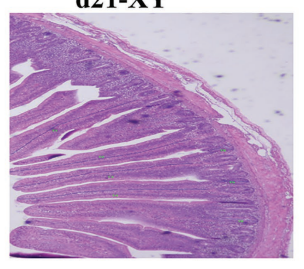

\section{d42-CON}

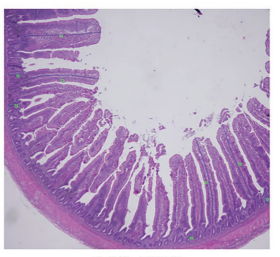

d42-XY

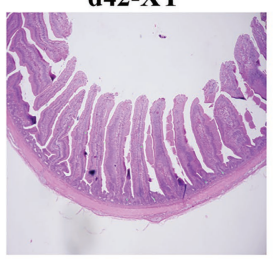

FIGURE 1 | Representative histological micrographs of ileum in broilers. CON is AT-xynA un-supplemented group; XY is 4,000 U/kg AT-xynA supplemented group.

TABLE 3 | Effects of AT-xynA on ileal morphology of broilers. ${ }^{1}$

\begin{tabular}{lccc}
\hline Items & CON & XY & Value of $\boldsymbol{p}$ \\
\hline day 7 & & & \\
Villus height, $\mu \mathrm{m}$ & $293.40 \pm 13.80$ & $359.20 \pm 13.94$ & 0.015 \\
Crypt depth, $\mu \mathrm{m}$ & $64.26 \pm 7.38$ & $71.86 \pm 11.19$ & 0.592 \\
Villus height to & $4.72 \pm 0.51$ & $5.37 \pm 0.79$ & 0.513 \\
crypt depth ratio & & & \\
day 14 & & & 0.097 \\
Villus height, $\mu \mathrm{m}$ & $428.80 \pm 18.56$ & $688.00 \pm 12.02$ & 0.104 \\
Crypt depth, $\mu \mathrm{m}$ & $85.82 \pm 7.75$ & $116.40 \pm 14.77$ & 0.331 \\
Villus height to & $5.12 \pm 0.39$ & $5.79 \pm 0.51$ & 0.010 \\
crypt depth ratio & & & 0.136 \\
day 21 & & & 0.930 \\
Villus height, $\mu \mathrm{m}$ & $492.70 \pm 16.23$ & $586.00 \pm 31.51$ & \\
Crypt depth, $\mu \mathrm{m}$ & $95.98 \pm 7.42$ & $113.70 \pm 1.25$ & 0.437 \\
Villus height to & $5.21 \pm 0.45$ & $5.16 \pm 0.21$ & \\
crypt depth ratio & & & \\
day 42 & & & \\
Villus height, $\mu \mathrm{m}$ & $792.40 \pm 19.21$ & $838.90 \pm 28.67$ & \\
Crypt depth, $\mu \mathrm{m}$ & $113.90 \pm 7.15$ & $110.10 \pm 5.34$ & \\
Villus height to & $7.08 \pm 0.52$ & $7.73 \pm 0.59$ & \\
crypt depth ratio & & & \\
\hline
\end{tabular}

${ }^{1}$ CON is AT-xynA un-supplemented group; XY is 4,000 U/kg AT-xynA supplemented group.Values are expressed as means \pm standard error of the mean (SEM), $n=6$.

Nocardiaceae $(p<0.01)$, Burkholderiaceae $(p=0.005)$, Staphylococcaceae $(p=0.013)$, Planococcaceae $(p=0.003)$, Peptococcaceae $(p=0.005)$, Thermoactinomycetaceae $(p=0.038)$, and Moraxellaceae $(p=0.003)$ was lower in ileal microbiota of broilers fed with AT-xynA.

At the genus level, as shown in Figure 5, the heatmap exhibited similarities and differences in bacterial community between two groups. In addition, differences in the composition of ileal microbiota at the genus level were further explored by the LEfSe method. The results indicated that compared with CON group, XY group was characterized by higher relative abundance of Lactobacillus ( $p<0.05$ ), lower abundance of Rothia, Pseudomonas, Aerococcus, Enterobacter, Microbacteroium, Staphylococcus, Exiguobacterium, Frisingicoccus, Glutamicibacter, Brachybacterium, Micrococcus, Leucobacter, Allorhizobium_Neorhizobium_Pararhizobium_ Rhizobiium, Monoglobus, Eubacterium_hallii_group, norank_f norank_o_Clostridia_vadinBB60_group, norank_f_norank_o_RF39, and Ruminococcus $(p<0.05)$ on day 21 . Compared with microbiota composition at genus level on day 42 , we also found that broilers from XY group had a higher proportion of Lactobacillus $(p<0.05)$ and lower proportion of Streptococcus, Bacillus, Enterococcus, UCG_005, Rhodococcus, Pseudogracilibacillus, and Burkholderia_ Caballeronia_Paraburkholderia $(p<0.05)$ than CON group.

\section{Predicted Functions of Microbial Communities in Ileum}

We utilized the PICRUSt algorithm to analyze KEGG pathways for predicting functions of intestinal microbiota related to nutrient metabolism. Four pathways associated with nutrient metabolism 
A

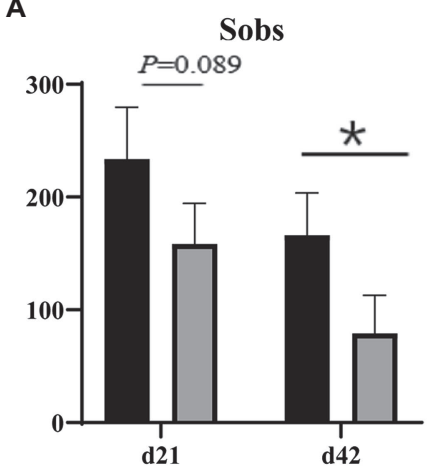

D

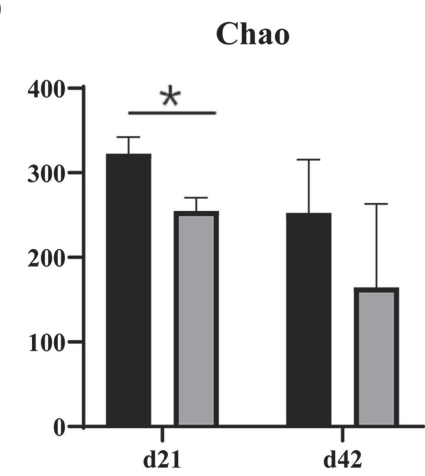

B

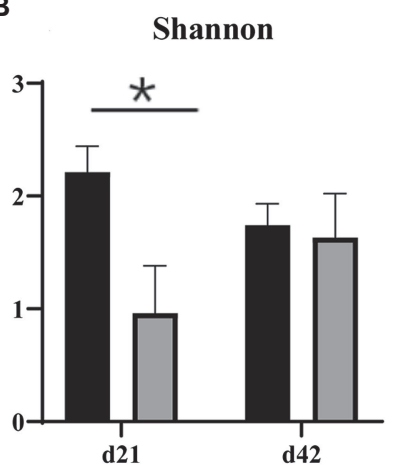

E

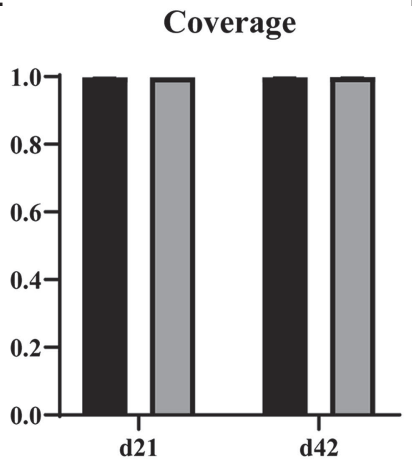

C
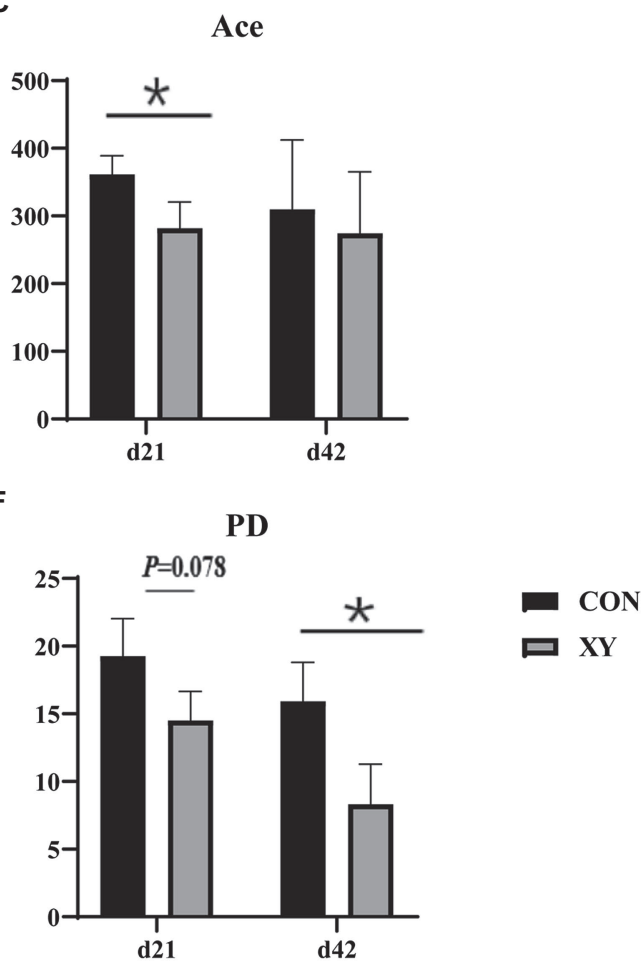

FIGURE 2 | Effects of AT-xynA on alpha diversity of ileal microbiota in broilers. (A) Observed richness (Sobs), (B) Shannon diversity index (Shannon), (C) ACE estimator (Ace) (D) Chao 1 estimator (Chao) (E) Good's coverage (Coverage), and (F) phylogenetic diversity (PD) of ileal microbiota in broilers on days 21 and 42. CON is AT-xynA un-supplemented group; XY is 4,000 U/kg AT-xynA supplemented group. The "*” indicates a significant difference between the two treatments $(p<0.05)$.

were analyzed including mineral absorption, carbohydrate digestion and absorption, butanoate metabolism, and starch and sucrose metabolism (Table 4). On day 21, the mineral absorption genes in XY group showed an increase $(p=0.046)$, whereas genes associated with carbohydrate digestion and absorption decreased in comparison with CON group $(p=0.034)$. On day 42 , the decrease of number of genes related to butanoate metabolism was observed in XY group $(p=0.008)$, and the number of genes involved in starch and sucrose metabolism displayed a trend to decrease in XY group $(p=0.081)$.

\section{Acetate and Lactate Concentrations in Ileal Digesta}

The lactate and acetate content in the ileal digesta of broilers are given in Table 5. Ileal samples of broilers from XY group showed a lower concentration of acetate than broilers fed CON diets on day 21 ( $p=0.039)$, but no differences in acetate content were found between the two groups on day 42 . No differences in lactate concentrations were observed between two treatments.

\section{Ileal Immune Factors and Antioxidant Activities}

We also analyzed immune factors and antioxidant activities in the ileum of broilers (Figure 6; Table 6). On day 42, the content of MDA displayed a decreasing trend in XY group $(p=0.085)$. In addition, there were no significant differences in the concentration of SOD between $\mathrm{CON}$ and $\mathrm{XY}$ groups. The concentrations of IL- $1 \beta$, IL- 6 , and TNF- $\alpha$ were profoundly modulated in the ileum of broilers. In ileal tissues from AT-xynA-fed broilers, the content of IL- $1 \beta$ showed a trend to decrease on day 7 ( $p=0.053)$. On day 14 , IL-6 level significantly decreased in the ileum of broilers from XY group $(p=0.015)$, and the content of TNF- $\alpha(p=0.076)$ and IL-1 $\beta(p=0.056)$ tended to decrease in broilers fed with AT-xynA. On day 21, no differences were observed in IL-1 $\beta$, IL- 6 , and TNF- $\alpha$ content in the ileum of broilers. On day 42, the ileal tissues of AT-xynA-fed broilers were marked by downregulation of IL-1 $\beta$ level ( $p=0.047)$, and the content of IL-6 showed a trend to decrease $(p=0.067)$.

\section{Ileal Barrier Functions}

The results showed ZO-1 and occludin gene expression levels in the ileum of broilers (Table 7). However, no significant differences were observed on $\mathrm{ZO}-1$ and occludin mRNA expression levels between two groups.

\section{Ileal Endocrine Peptides}

The effects of AT-xynA in wheat-based diets on endocrine peptide content are presented in Table 8. These results showed 
A

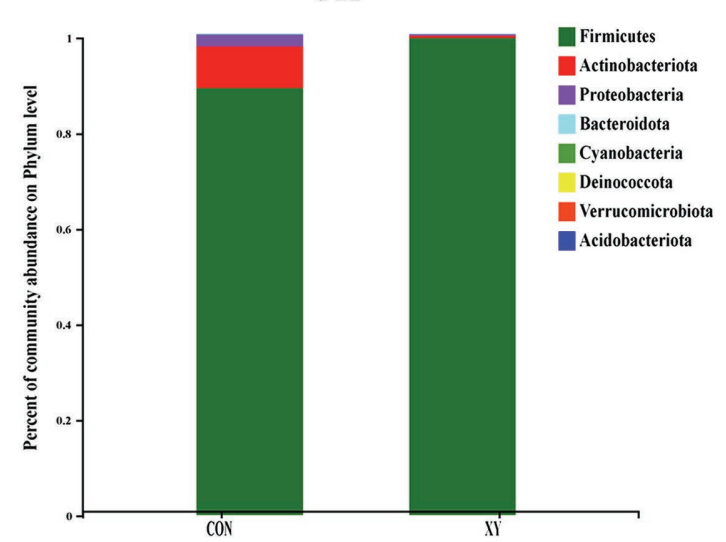

C

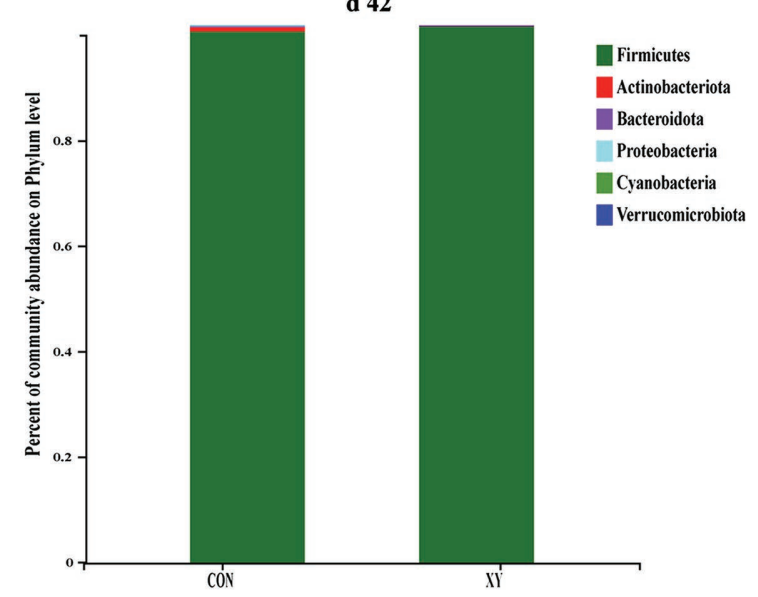

B

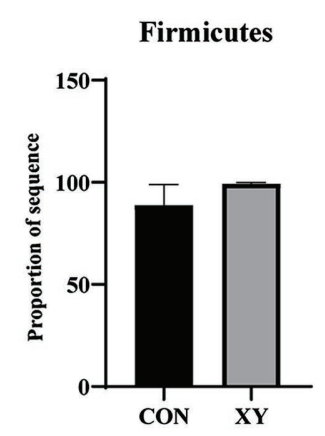

d 21

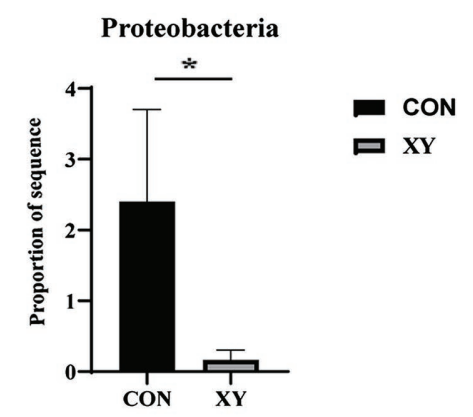

D

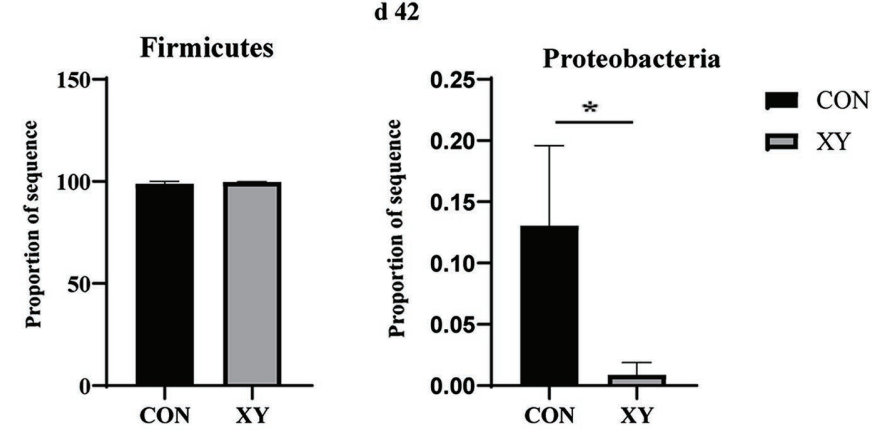

FIGURE 3 | Effects of AT-xynA on ileal microbiota composition at the phylum level. (A,C) lleal microbiota composition at the phylum level in broilers; (B,D) alterations of the abundance of bacterial phyla found in the ileum of broilers. CON is AT-xynA un-supplemented group; XY is 4,000 U/kg AT-xynA supplemented group. The "*” indicates a significant difference between the two treatments $(p<0.05)$.

that concentrations of GLP-1 $(p=0.098)$ and IGF-1 $(p=0.03)$ were positively affected by xylanase supplementation on day 7. On day 21, IGF-1 concentration in the ileum of broilers fed with diets containing AT-xynA showed a trend to increase compared with CON group $(p=0.075)$.

\section{Correlations Between lleal Microbiota, Lactate and Acetate Levels, Morphology, and Immune Factors}

The correlations between ileal microbiota, metabolites, morphology, and cytokine levels were evaluated by the spearman correlation analysis (Figure 7). The concentration of lactate was positively associated with the abundance of Lactobacillus and negatively associated with the abundance of Pseudomonas, Brachybacterium, unclassified_f_Lachnospiraceae, Enterobacter, Streptococcus, Marvinbryantia, Sellimonas, Blautia, Ruminococcus_torques_group, Stenotrophomonas, and Acinetobacter $(p<0.05)$. The intestinal development (higher $\mathrm{VH}$, lower $\mathrm{CD}$, and higher VH/CD suggesting greater intestinal development) exhibited a positive correlation with the abundance of Lactobacillus and a negative correlation with Enterococcus, Staphylococcus, Pseudomonas, Aerococcus, Microbacterium, Rothia, Candidatus_Arthromitus, Enterobacter, Streptococcus, Marvinbryantia, Sellimonas, Blautia, Ruminococcus torques_group, Stenotrophomonas, and Acinetobacter $(p<0.05)$. The immune functions (lower levels of IL- $1 \beta$, IL- 6 , and TNF- $\alpha$ indicating greater immune functions) displayed a negative correlation with the abundance of Legionella, Enterococcus, Staphylococcus, Pseudomonas, and Candidatus_Arthromitus $(p<0.05)$.

\section{DISCUSSION}

In the current study, AT-xynA supplementation could improve broilers growth performance, and this observation was anticipated and consistent with published reports. González-Ortiz et al. (2016) demonstrated that supplementing xylanase to wheatbased diets could numerically increase body weight gain (BWG) and FCR of broilers. Zhang et al. (2014) also showed improved 


\section{A}

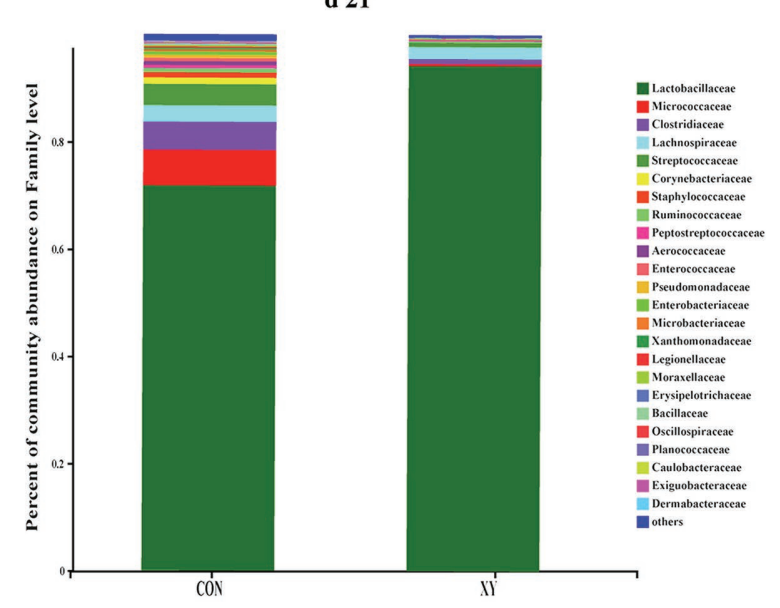

C

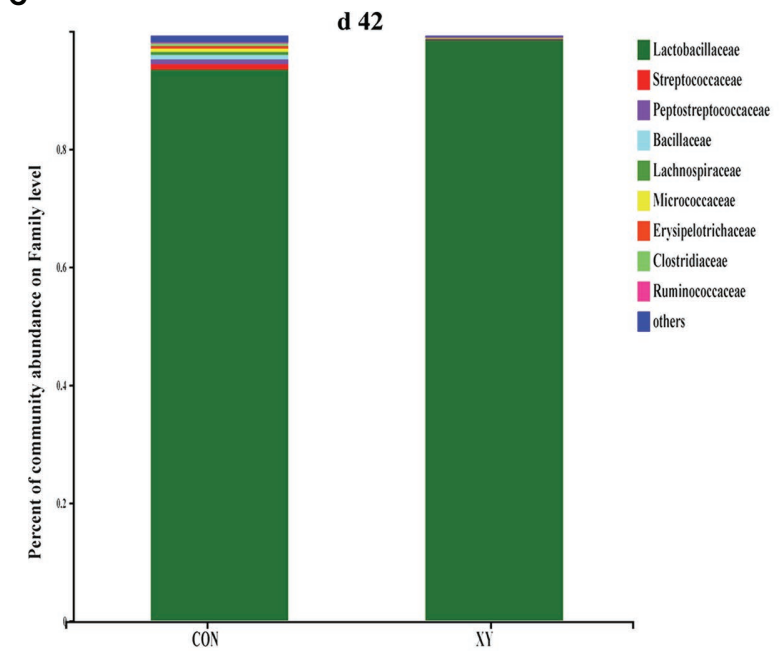

B

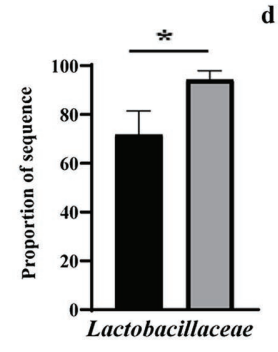

d 21

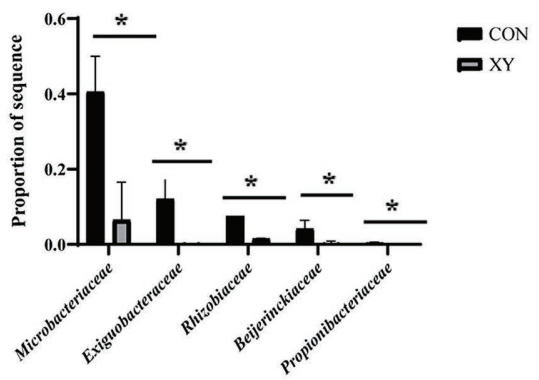

D

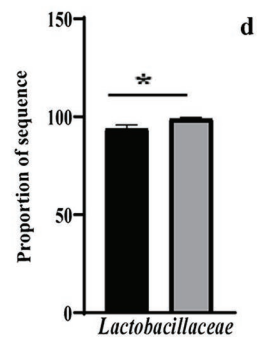

d 42

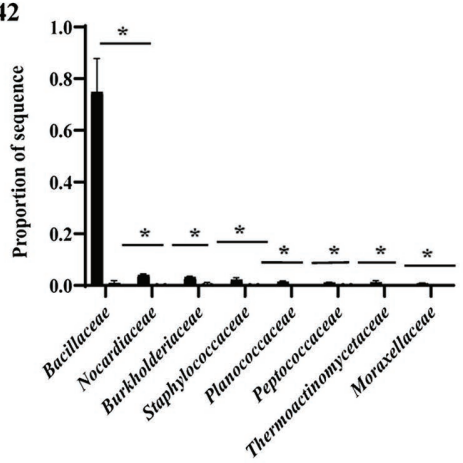

FIGURE 4 | Effects of AT-xynA on ileal microbiota composition at the family level. (A,C) lleal microbiota composition at the family level with the relative abundance higher than $0.1 \%$ in broilers; (B,D) alterations of the abundance of bacterial families found in the ileum of broilers. CON is AT-xynA un-supplemented group; XY is 4,000 U/kg AT-xynA supplemented group. The "*” indicates a significant difference between the two treatments $(p<0.05)$.

FCR and BWG of broilers fed wheat-based diets supplemented xylanases. However, positive effects of AT-xynA on growth performance of broilers were not observed until the third week of age after hatch, indicated by increased ADG from days 14-21 and days 21-42. It has demonstrated that the development of digestive system of chicks occurs rapidly in the first few days after hatching, and nutrients were utilized preferentially for the gastrointestinal tract development of chicks relative to BWG (Lji et al., 2001).

The process of early intestinal growth and development involves changes in intestinal morphology, and the enhanced intestinal growth could efficiently improve absorption ability and nutrient utilization, and may further benefit to the subsequent growth performance (Dai et al., 2020). We found that the addition of AT-xynA could improve ileal $\mathrm{VH}$, and the improvement in $\mathrm{VH}$ might be related to the greater absorption surface and faster epithelial cells turnover, suggesting improvement of nutrient absorption capacity in the intestine (Li et al., 2019). Similarity, Wu et al. (2004a) suggested that the addition of xylanase to wheat-based diets increased $\mathrm{VH}$ in the ileum of broilers on day 21. Luo et al. (2009) also reported that xylanase could increase ileal VH and $\mathrm{VH} / \mathrm{CD}$ ratio in broilers fed wheat-based diets. Dietary NSPs could negatively influence epithelial cell turnover of the intestine, but degradation of xylans might be able to alleviate these negative effects on intestinal morphology in broilers (Lee et al., 2020). However, some reports showed that xylanase supplementation had no effects on VH, CD, and $\mathrm{VH} / \mathrm{CD}$ ratio of the ileum in broilers, and we hypothesized that the type of wheat, proportion of dietary wheat, characteristics of xylanase, and age of broilers might be the reason why broilers showed different responses to xylanase supplementation (Wu et al., 2004b). In fact, no significant differences in ileal morphology of broilers were found on 


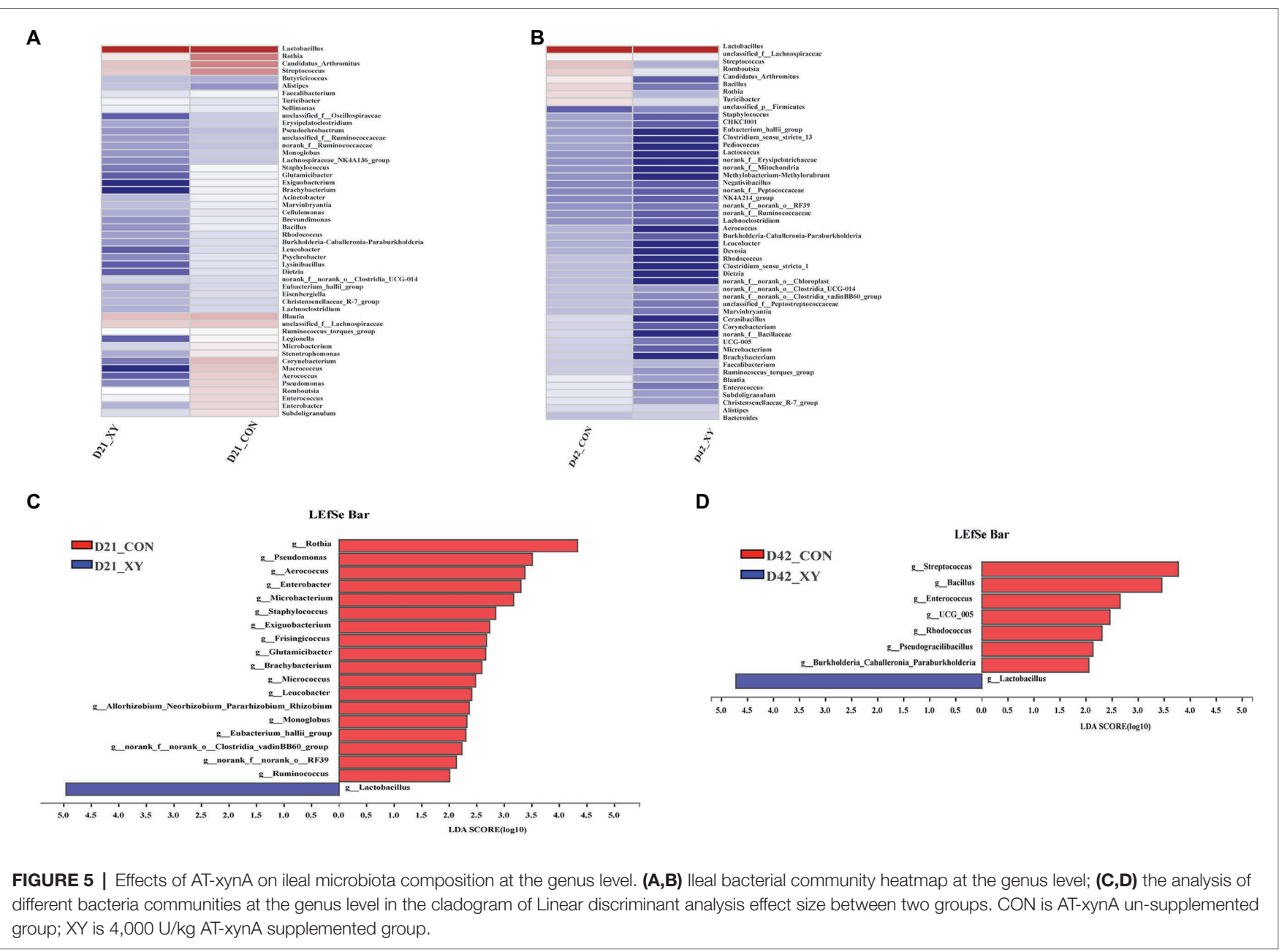

day 42 in the current study. This may be explained by the development of intestine in broilers, and the morphological maturation of the small intestine occurs during the first week (Lji et al., 2001). Thus, young broilers might be particularly sensitive to NSP, and benefits of xylanase in young broilers are greater.

In addition to direct effects of gut physiology and morphology by viscous NSPs, the negative effects of soluble NSPs on broilers performance appear to be indirectly associated with changes of intestinal microbiota composition. The type of diets and dietary additives has been reported to be able to affect the composition of intestinal microbiota in broilers (Sudhir and Jha, 2019). The alpha diversity of broilers intestinal microbiota could reflect richness and evenness of microbial community (Wagner et al., 2018). In this study, the alpha diversity of ileal microbiota obviously decreased with xylanase supplementation. Hübener et al. (2002) demonstrated that xylanase exhibited a reduction in the total microbial load in terms of bacteria counts in the small intestine. The broilers fed wheat-based diets resulted in the increase of digesta viscosity, and the viscous digesta could reduce intestinal mobility and decrease oxygen tension, thus stimulated the colonization of certain bacteria in the ileum, particularly pathogenic microbiota (Hübener et al., 2002).
Moreover, undigested protein and starch in the small intestine might result in the bacterial overgrowth (Bedford, 2018). Supplementing xylanase to wheat-based diets could decrease the amount of nutrients which are available as substrates utilized by bacteria in the ileum, thus reduced the number of bacteria in the small intestine (Choct et al., 1999; Apajalahti et al., 2004).

At the phylum level, similar to previous reports, Firmicutes was dominant in the ileum of broilers (Rychlik, 2020). On days 21 and 42, the abundance of Proteobacteria was lower in broilers supplemented xylanase. It has been demonstrated that many human pathogens, such as Escherichia, Campylobacter, Salmonella, Helicobacter, and Pseudomonas, were found in Proteobacteria phylum (Rizzatti et al., 2017). Thus, the enhanced abundance of Proteobacteria might be a microbial signature for diseases. The current study demonstrated that the abundance of Lactobacillaceae (belonging mainly to the genus Lactobacillus) in the ileum was promoted by xylanase supplementation. Engberg et al. (2004) also reported that xylanase supplementation could increase the abundance of Lactobacillus in the ileum of broilers fed wheat-based diets. Lactobacillus spp. is beneficial to the gut health of broilers due to probiotic effects; it could lead to the competitive exclusion of the pathogenic 
TABLE 4 | Prediction on metabolism of ileal bacterial communities using PICRUSt analysis. ${ }^{1}$

\begin{tabular}{|c|c|c|c|}
\hline Items & CON & $\mathbf{X Y}$ & Value of $p$ \\
\hline \multicolumn{4}{|l|}{ day 21} \\
\hline Mineral absorption & $50.67 \pm 3.89$ & $435.30 \pm 26.60$ & 0.046 \\
\hline $\begin{array}{l}\text { Carbohydrate } \\
\text { digestion and } \\
\text { absorption }\end{array}$ & $3929.7 \pm 263.4$ & $1330.0 \pm 184.9$ & 0.034 \\
\hline $\begin{array}{l}\text { Butanoate } \\
\text { metabolism }\end{array}$ & $104658 \pm 1374$ & $112353 \pm 2842$ & 0.693 \\
\hline $\begin{array}{l}\text { Starch and } \\
\text { sucrose } \\
\text { metabolism }\end{array}$ & $137024 \pm 1714$ & $118300 \pm 2605$ & 0.185 \\
\hline \multicolumn{4}{|l|}{ day 42} \\
\hline Mineral absorption & $4.00 \pm 0.08$ & $73.33 \pm 15.10$ & 0.011 \\
\hline $\begin{array}{l}\text { Carbohydrate } \\
\text { digestion and } \\
\text { absorption }\end{array}$ & $2694.0 \pm 123.5$ & $719.7 \pm 83.8$ & 0.100 \\
\hline $\begin{array}{l}\text { Butanoate } \\
\text { metabolism }\end{array}$ & $89377 \pm 1545$ & $58082 \pm 1323$ & 0.008 \\
\hline $\begin{array}{l}\text { Starch and } \\
\text { sucrose } \\
\text { metabolism }\end{array}$ & $118128 \pm 3171$ & $92262 \pm 1704$ & 0.081 \\
\hline
\end{tabular}

${ }^{1}$ CON is AT-xynA un-supplemented group; XY is 4,000 U/kg AT-xynA supplemented group. Values are expressed as means $\pm S E M, n=6$.

TABLE 5 | Effects of AT-xynA on lactate and acetate concentrations in ileal digesta of broilers $(\mathrm{mg} / \mathrm{kg}){ }^{1}$

\begin{tabular}{lccc}
\hline Items & CON & XY & Value of $\boldsymbol{p}$ \\
\hline day 21 & & & \\
Lactate & $1029.20 \pm 49.88$ & $1174.70 \pm 155.60$ & 0.423 \\
Acetate & $531.40 \pm 20.66$ & $311.10 \pm 32.29$ & 0.039 \\
day 42 & & & \\
Lactate & $1992.60 \pm 121.30$ & $3054.20 \pm 180.20$ & 0.293 \\
Acetate & $424.70 \pm 24.35$ & $595.40 \pm 48.20$ & 0.411 \\
\hline
\end{tabular}

${ }^{1}$ CON is AT-xynA un-supplemented group; $X Y$ is 4,000 U/kg AT-xynA supplemented group. Values are expressed as means $\pm S E M, n=6$.

bacteria (Gao et al., 2017). In the current study, we found that Lactobacillus enrichment was positively correlated with lactate concentration and mucosal $\mathrm{VH}$ in the ileum of broilers. The previous study also reported that the abundance of Lactobacillus was positively related to ileal $\mathrm{VH}$ in layer chicks (Dai et al., 2020).

On day 21, the abundance of genus Rothia, Glutamicibacter, and Micrococcus (belonging to the family Micrococcaceae) was higher in broilers fed wheat-based diets un-supplemented xylanase. The genus Rothia has been reported to be able to result in serious infections, particularly in immunocompromised hosts (Ramanan et al., 2014). The current study also showed that Rothia was negatively associated with the intestinal development. However, the knowledge about metabolism and role of genus Glutamicibacter, Micrococcus, and family Micrococcaceae was limited. Higher abundance of genus Microbacterium, Enterobacter, Staphylococcus, and Pseudomonas in the ileum was also observed in broilers fed $\mathrm{CON}$ diets. Strains belonging to the genus
Microbacterium (within the family Microbacteriaceae), genus Enterobacter (within the family Enterobacteriaceae), genus Staphylococcus (within the family Staphylococcaceae), and genus Pseudomonas (within the family Pseudomonadaceae) have recently been identified as pathogenic bacteria and might be related to a wide range of infections (Funke et al., 1997; Hotterbeekx et al., 2016). We also found that the abundance of Enterobacter, Staphylococcus, and Pseudomonas was negatively correlated with lactate concentration, development of intestinal morphology, and ileal immune function. On day 42, remarkable increase in the abundance of pathogenic bacteria, such as family Moraxellaceae, Nocardiaceae, Burkholderiaceae, Staphylococcaceae, genus Streptococcus, and Enterococcus, was shown in broilers from CON group; these bacteria contain a few animal pathogens which might result in a wide variety of infections (Arana et al., 2001; Chadfield et al., 2005; Neveling et al., 2017; Chen et al., 2019; DiCenzo et al., 2019). The higher abundance of pathogenic bacteria might explain poor performance of broilers from CON group. In addition, higher abundance of genus Frisingicoccus, Eubacterium_hallii_group, and Ruminococcus was observed in xylanase un-supplemented broilers on day 21, and higher abundance of Peptococcaceae and Bacillaceae was shown in broilers from CON group on day 42. These bacteria belong to phylum Firmicutes; however, this phylum is thought to contribute to the production of SCFA (Dai et al., 2021). Supplementing xylanase could also decrease the abundance of family Propionibacteriaceae on day 21 , and bacteria belonging to family Propionibacteriaceae could also promote the production of SCFA. Choct et al. (1996) have reported that supplementing soluble NSPs to broilers diets could elevate fermentation in the small intestine. The increase in the abundance of SCFA-producing bacteria might be related to the alleviation of excess fermentation due to xylanase.

In PICRUSt function analysis, the ileal microbiota of broilers supplemented xylanases showed an increase in mineral absorption pathway. This result demonstrated that broilers from XY group could have a higher capacity to utilize minerals. However, bacterial carbohydrate digestion and absorption, butanoate metabolism, and starch and sucrose metabolism pathways were higher in CON group. The upregulation in these pathways might be due to high NSP content present in broilers provided wheat-based diets un-supplemented xylanase. The nutrients in the ileum of broilers fed diets un-supplemented enzymes were exposed; however, nutrients had little chance to contact digestive enzymes in the small intestine due to high digesta viscosity, and undigested nutrients were fermented by microbiome to produce SCFA. In this study, we also have found that bacterial fatty acid production increased in the ileum of broilers from CON group. Supplementing NSP-degrading enzymes to diets also has been reported to improve ileal starch digestibility of broilers (Choct et al., 1996). Given that nutrient digestion and absorption capacity significantly increased in XY group, lower carbohydrate digestion and absorption, and starch and sucrose metabolism pathways in XY group were likely due to less nutrients present in ileal content for bacteria to utilize and hence a compensatory upregulation in metabolic pathways. 
A

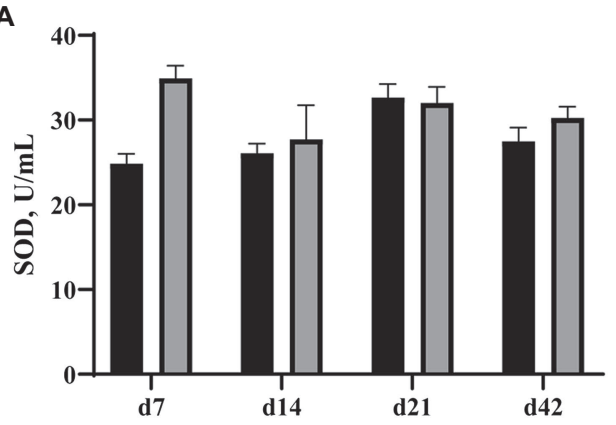

B

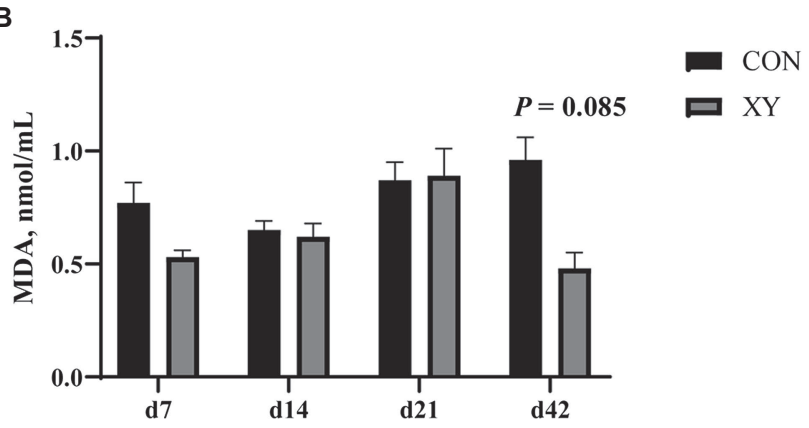

FIGURE 6 | Effects of AT-xynA on antioxidant capacities in the ileum of broilers. (A) SOD, superoxide dismutase; (B) MDA, malondialdehyde concentrations in the ileum of broilers. CON is AT-xynA un-supplemented group; XY is 4,000 U/kg AT-xynA supplemented group.

TABLE 6 | Effects of AT-xynA on ileal immune function of broilers. ${ }^{1}$

\begin{tabular}{|c|c|c|c|}
\hline Items & CON & $X Y$ & Value of $p$ \\
\hline \multicolumn{4}{|l|}{ day 7} \\
\hline $\begin{array}{l}\text { Interleukin- } 1 \beta, \mathrm{pg} . / \mathrm{mg} \\
\text { Interleukin- } 6, \mathrm{ng} / \mathrm{g} \\
\text { Interleukin-10, pg./mg } \\
\text { Tumor necrosis factor- } \alpha, \mathrm{ng} / \mathrm{g} \\
\text { Interferon- } \gamma, \mathrm{pg} . / \mathrm{mg}\end{array}$ & $\begin{array}{r}30.94 \pm 2.02 \\
11.25 \pm 1.40 \\
3.42 \pm 0.23 \\
34.47 \pm 3.85 \\
3.32 \pm 0.11\end{array}$ & $\begin{array}{r}23.37 \pm 1.91 \\
9.09 \pm 0.19 \\
3.85 \pm 0.63 \\
29.90 \pm 4.17 \\
3.30 \pm 0.58\end{array}$ & $\begin{array}{l}0.053 \\
0.262 \\
0.553 \\
0.466 \\
0.978\end{array}$ \\
\hline \multicolumn{4}{|l|}{ day 14} \\
\hline $\begin{array}{l}\text { Interleukin- } 1 \beta, \mathrm{pg} . / \mathrm{mg} \\
\text { Interleukin- } 6, \mathrm{ng} / \mathrm{g} \\
\text { Interleukin-10, pg./mg } \\
\text { Tumor necrosis factor- } \alpha, \mathrm{ng} / \mathrm{g} \\
\text { Interferon- } \gamma, \mathrm{pg} . / \mathrm{mg}\end{array}$ & $\begin{array}{r}29.24 \pm 4.05 \\
14.57 \pm 1.22 \\
4.23 \pm 0.56 \\
40.38 \pm 5.32 \\
4.54 \pm 0.70\end{array}$ & $\begin{array}{r}18.16 \pm 0.90 \\
9.27 \pm 0.45 \\
3.17 \pm 0.25 \\
27.15 \pm 1.57 \\
3.72 \pm 0.24\end{array}$ & $\begin{array}{l}0.056 \\
0.015 \\
0.155 \\
0.076 \\
0.329\end{array}$ \\
\hline \multicolumn{4}{|l|}{ day 21} \\
\hline $\begin{array}{l}\text { Interleukin-1 } \beta, \mathrm{pg} . / \mathrm{mg} \\
\text { Interleukin- } 6, \mathrm{ng} / \mathrm{g} \\
\text { Interleukin-10, pg./mg } \\
\text { Tumor necrosis factor- } \alpha, \mathrm{ng} / \mathrm{g} \\
\text { Interferon- } \gamma, \mathrm{pg} . / \mathrm{mg}\end{array}$ & $\begin{array}{r}42.54 \pm 5.60 \\
13.33 \pm 2.59 \\
4.28 \pm 0.29 \\
37.87 \pm 2.79 \\
3.96 \pm 0.22\end{array}$ & $\begin{aligned} 30.27 & \pm 1.92 \\
9.28 & \pm 0.19 \\
4.63 & \pm 1.32 \\
36.97 & \pm 2.63 \\
4.31 & \pm 0.97\end{aligned}$ & $\begin{array}{l}0.107 \\
0.258 \\
0.811 \\
0.938 \\
0.740\end{array}$ \\
\hline \multicolumn{4}{|l|}{ day 42} \\
\hline $\begin{array}{l}\text { Interleukin-1 } \beta, \mathrm{pg} . / \mathrm{mg} \\
\text { Interleukin- } 6, \mathrm{ng} / \mathrm{g} \\
\text { Interleukin-10, pg./mg } \\
\text { Tumor necrosis factor- } \alpha, \mathrm{ng} / \mathrm{g} \\
\text { Interferon- } \gamma, \mathrm{pg} . / \mathrm{mg}\end{array}$ & $\begin{aligned} 43.17 & \pm 3.51 \\
12.31 & \pm 1.07 \\
4.09 & \pm 0.86 \\
49.41 & \pm 1.10 \\
3.87 & \pm 0.84\end{aligned}$ & $\begin{aligned} 29.37 & \pm 3.38 \\
8.62 & \pm 1.03 \\
4.57 & \pm 1.13 \\
25.32 & \pm 3.58 \\
4.33 & \pm 0.83\end{aligned}$ & $\begin{array}{l}0.047 \\
0.067 \\
0.753 \\
0.108 \\
0.716\end{array}$ \\
\hline
\end{tabular}

${ }^{1}$ CON is AT-xynA un-supplemented group; XY is 4,000 U/kg AT-xynA supplemented group. Values are expressed as means $\pm S E M, n=6$.

Lower concentration of acetate was observed in broilers fed xylanase in the current study. Choct et al. (1996) demonstrated that broilers fed high NSP diets showed higher concentrations of SCFA in the ileum compared with broilers fed enzymesupplemented diets. Józefiak et al. (2007) also reported that adding xylanase to broilers rye-based diets could decrease SCFA concentrations in the ileum. The addition of xylanases could effectively reduce the amount of substrates available for the colonization of fermentative bacteria, and the reduction in microbial fermentation could decrease the production of SCFA and alleviate excess fermentation in the ileum (Choct et al., 1999). Higher abundance of Lactobacillus was observed in broilers fed xylanase; however, no differences in lactate concentrations were observed
TABLE 7 | Effects of AT-xynA on relative mRNA expression of ileal tight junction genes in broilers. ${ }^{1}$

\begin{tabular}{lccc}
\hline Items & CON & XY & Value of $\boldsymbol{p}$ \\
\hline day $\mathbf{7}$ & & & \\
ZO-1 & & & \\
Occludin & $1.00 \pm 0.18$ & $0.80 \pm 0.01$ & 0.584 \\
day 14 & $1.00 \pm 0.17$ & $0.74 \pm 0.06$ & 0.458 \\
ZO-1 & & & \\
Occludin & $1.00 \pm 0.13$ & $1.41 \pm 0.14$ & 0.169 \\
day 21 & $1.00 \pm 0.14$ & $1.40 \pm 0.11$ & 0.162 \\
ZO-1 & & & \\
Occludin & $1.00 \pm 0.14$ & $1.02 \pm 0.12$ & 0.881 \\
day 42 & $1.00 \pm 0.12$ & $1.00 \pm 0.09$ & 0.937 \\
ZO-1 & & & 0.323 \\
Occludin & $1.00 \pm 0.09$ & $1.11 \pm 0.05$ & 0.438 \\
\hline
\end{tabular}

${ }^{1} \mathrm{CON}$ is AT-xynA un-supplemented group; XY is 4,000 U/kg AT-xynA supplemented group. Values are expressed as means $\pm S E M, n=6$. The values were calculated relative to the expression of $\beta$-actin with formula $2^{-\Delta \Delta C t}$. ZO-1, zonula occludens- 1 .

in broilers from the two groups. We hypothesized that lactate produced by Lactobacillus was absorbed in the intestine or used as a substrate for lactate-utilizing bacteria.

It has been reported that the immune system could be affected by providing feed additives to diets that could influence the composition of intestinal microbiota (Liu et al., 2012; Schokker et al., 2017). We found the reduction in IL-1 $\beta$ and TNF- $\alpha$ concentrations of ileal tissues in broilers from $\mathrm{XY}$ group. IL- $1 \beta$ and TNF- $\alpha$ are important proinflammatory cytokines that produced and released by macrophages, and they are considered to regulate pathological responses that occur in inflammatory conditions (Jensen et al., 2020). Zhang et al. (2018) demonstrated that supplementing xylanases and fermented polysaccharide of Hericium caputmedusae (FPHC) increased the anti-inflammatory capacity of broilers by improving serum IL- $1 \alpha$ and IL-10, and decreasing IL- $1 \beta$ and TNF- $\alpha$ concentrations. Wang et al. (2021) reported that the exogenous enzyme complex (primarily contained 3,200 U/ $\mathrm{kg} \beta$-glucanase and $6,225 \mathrm{U} / \mathrm{kg}$ xylanase) could significantly increase the levels of IL- 6 and IL-10, and decrease IL- $1 \beta$ and TNF- $\alpha$ levels in the jejunum of broilers. Reduction in 
TABLE 8 | Effects of AT-xynA on ileal endocrine peptide concentrations of broilers. $^{1}$

\begin{tabular}{|c|c|c|c|}
\hline Items & CON & $X Y$ & $\begin{array}{c}\text { Value } \\
\text { of } p\end{array}$ \\
\hline \multicolumn{4}{|l|}{ day 7} \\
\hline $\begin{array}{l}\text { Epidermal growth factor, pg./mg } \\
\text { Glucagon-like peptide-1, pmol/g } \\
\text { Glucagon-like peptide-2, pg./mg } \\
\text { Insulin-like growth factor-1, ng/mg }\end{array}$ & $\begin{aligned} 23.45 & \pm 3.02 \\
0.69 & \pm 0.04 \\
200.70 & \pm 26.26 \\
17.43 & \pm 1.36\end{aligned}$ & $\begin{aligned} 18.10 & \pm 0.26 \\
0.89 & \pm 0.08 \\
156.50 & \pm 7.41 \\
21.95 & \pm 1.53\end{aligned}$ & $\begin{array}{l}0.217 \\
0.098 \\
0.181 \\
0.093\end{array}$ \\
\hline \multicolumn{4}{|l|}{ day 14} \\
\hline $\begin{array}{l}\text { Epidermal growth factor, pg./mg } \\
\text { Glucagon-like peptide-1, pmol/g } \\
\text { Glucagon-like peptide-2, pg./mg } \\
\text { Insulin-like growth factor-1, ng/mg }\end{array}$ & $\begin{array}{r}21.37 \pm 2.27 \\
0.71 \pm 0.02 \\
153.20 \pm 4.77 \\
17.17 \pm 0.19\end{array}$ & $\begin{aligned} 18.66 & \pm 1.98 \\
0.99 & \pm 0.12 \\
153.20 & \pm 22.33 \\
22.39 & \pm 2.34\end{aligned}$ & $\begin{array}{l}0.420 \\
0.141 \\
1.000 \\
0.157\end{array}$ \\
\hline \multicolumn{4}{|l|}{ day 21} \\
\hline $\begin{array}{l}\text { Epidermal growth factor, pg./mg } \\
\text { Glucagon-like peptide-1, pmol/g } \\
\text { Glucagon-like peptide-2, pg./mg } \\
\text { Insulin-like growth factor-1, ng/mg }\end{array}$ & $\begin{aligned} 25.34 & \pm 1.36 \\
0.73 & \pm 0.02 \\
201.70 & \pm 12.91 \\
16.87 & \pm 1.45\end{aligned}$ & $\begin{aligned} 24.57 & \pm 7.26 \\
1.12 & \pm 0.15 \\
213.10 & \pm 12.73 \\
28.68 & \pm 4.72\end{aligned}$ & $\begin{array}{l}0.926 \\
0.118 \\
0.843 \\
0.075\end{array}$ \\
\hline \multicolumn{4}{|l|}{ day 42} \\
\hline $\begin{array}{l}\text { Epidermal growth factor, pg./mg } \\
\text { Glucagon-like peptide-1, pmol/g } \\
\text { Glucagon-like peptide-2, pg./mg } \\
\text { Insulin-like growth factor-1, ng/mg }\end{array}$ & $\begin{array}{r}21.21 \pm 7.87 \\
1.08 \pm 0.34 \\
181.70 \pm 6.71 \\
21.17 \pm 7.84\end{array}$ & $\begin{array}{r}24.53 \pm 2.00 \\
0.88 \pm 0.09 \\
194.70 \pm 4.87 \\
22.24 \pm 1.71\end{array}$ & $\begin{array}{l}0.703 \\
0.600 \\
0.853 \\
0.905\end{array}$ \\
\hline
\end{tabular}

${ }^{1}$ CON is AT-xynA un-supplemented group; XY is 4,000 U/kg AT-xynA supplemented group. Values are expressed as means $\pm S E M, n=6$.

the serum TNF- $\alpha$ content in nursey pigs fed diets with corn distiller's dried grains was also observed (Chen et al., 2020). Similar to IL-1 $\beta$, IL-6 is another pro-inflammatory cytokine, and it responses to immune stimulation (Umare et al., 2014). It has been widely accepted that nutrition could impact immune function, and deficiency in nutrients could negatively affect normal immune responses (Wu et al., 2018). Thus, the addition of xylanases could promote the digestion and absorption of nutrients by degrading arabinoxylans, which in turn may contribute to the improvement of immune function. Moreover, extensive interactions occur between intestinal microbiota and immune system of the host (Sudhir and Jha, 2019). We have observed that the addition of xylanase stimulated the colonization of beneficial bacteria and reduced the abundance of harmful bacteria in the intestine of broilers. Therefore, the results suggested that xylanase might modulate immune responses in broilers by regulation of intestinal microbiome that positively affects nutrient digestion and utilization along with positively affects intestinal immunity. At the same time, we observed that effects of xylanase on immunity function were greater at young age. After hatch, the development of immune system and colonization of intestinal microbiota occur rapidly; in addition, the microbiota colonization in broilers is essential for this development (Bar-Shira et al., 2003). Thus, we hypothesized that older broilers had a greater capacity to deal with negative effects of NSP due to mature gut microbiota and immune system.

Oxidative stress is essential to the intestinal health of broilers; MDA as one of the essential toxic lipid peroxides could cause damage to the membrane structure and function (Gawe et al., 2004). In this study, these results suggested that xylanase supplementation could enhance the antioxidant capacity of broilers. Zhang et al. (2018) has demonstrated that xylanase and FPHC treatment reduced the level of serum MDA in broilers. Wang et al. (2021) reported that the exogenous enzyme complex (primarily contained 3,200 U/kg $\beta$-glucanase and $6,225 \mathrm{U} / \mathrm{kg}$ xylanase) could improve the activities of T-AOC, SOD, and GSH-Px in the jejunal mucosa of broilers. The application of xylanase could also effectively reduce the content of MDA in the mucosa of jejunum in newly weaned piglets (Duarte et al., 2019). Petry et al. (2020) reported that xylanase supplementation improved the antioxidant capacity in the ileum of growing pigs provided insoluble corn-based fiber. However, it is still unclear how xylanase could reduce oxidative stress and improve antioxidant capacity of broilers. Duarte et al. (2019) thought that negative effects of NSPs toward digesta could influence molecules in the intestinal cell wall, and this may be the reason why xylanase could decrease oxidative stress.

The intestinal epithelial barrier function is the first line of defense against intruders of the body, and intestinal barrier dysfunction might result in the loss of integrity of the epithelium and an increase risk of gastrointestinal diseases (Camilleri et al., 2012). The barrier contains several unique proteins; occludin and ZO-1 are two essential components in the modulation of barrier function of the intestine (Fanning et al., 1998). In the current study, no significant differences were observed in occludin and ZO-1 mRNA expression levels in the ileum of broilers from two groups. However, Petry et al. (2020) suggested that supplementing xylanase could upregulate claudin-4 and occludin mRNA expression level in the ileum of growing pigs fed insoluble corn-based fiber. Tiwari et al. (2018) also reported that xylanase supplementation increased claudin, occludin, and ZO-1 mRNA expression in the jejunum of nursey pigs fed corn distiller's dried grain with soluble based diets. In this study, no differences in the intestinal barrier function were observed, and one potential speculation could be that the content of NSP which caused barrier function damage was insufficient.

L-cells as important component of the gut-brain axis are primarily located in the jejunum and ileum of chickens; the endocrine peptides secreted from L-cells are essential to the digestion of nutrients and development of the intestine (Lim et al., 2009). Higher levels of GLP-1 and IGF-1 were observed in the ileum of broilers from XY group. Glucagonlike peptide- 1 has been demonstrated to play a crucial pole in gastric emptying reduction and small intestine growth rate (Lim et al., 2009). As a result of the increase of GLP-1 concentration, the nutrients could be more efficiently digested and absorbed. Gao et al. (2007) also reported that supplementing xylanase could increase blood IGF-1 content in 21-day-old cockerels. Insulin-like growth factor-1 also benefits to the growth rate and energy metabolism; this endocrine peptide is beneficial to the growth and function of almost every organ (Wang et al., 2017). Insulin-like growth factor-1 has been reported to increase $\mathrm{VH}$ in rats 


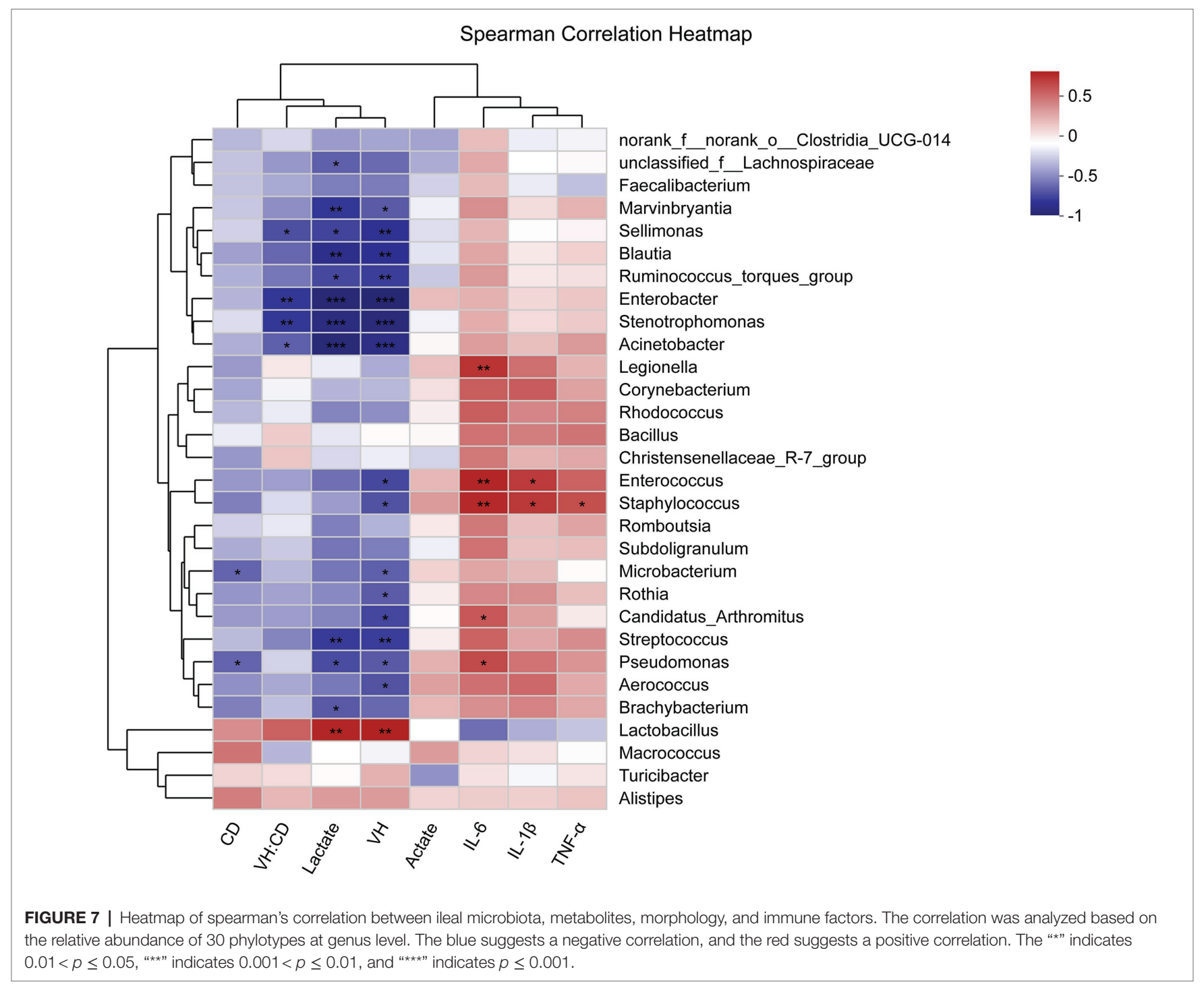

(Mantell et al., 1995); thus, enhanced IGF-1 content in broilers supplemented xylanase might partly explain why improvement in ileal morphology was observed. However, we only observed that the content of IGF-1 and GLP-1 in $\mathrm{XY}$ group was higher than that in CON group on days 7 and 21. The intestine microbiota of older broilers might be more mature and stable, the ability of microbiota to degrade arabinoxylans grew, and thus, xylanase supplementation had a weak positive effect on intestinal health (Bautil et al., 2019). This might suggest that the effects of xylanase are more pronounced in young broilers due to their low digestive capacity to wheat-based diets.

\section{CONCLUSION}

Xylanase supplementation to broiler wheat-based diets had beneficial effects on ileal microbiota composition, which was reflected in enhanced relative abundance of Lactobacillus, and reduced relative abundance of potentially pathogenic bacteria. Xylanase supplementation decreased the abundance of SCFA-producing bacteria and acetate concentration in the ileum; this reduction suggested that xylanase could alleviate excess fermentation of bacteria. In addition, AT-xynA could improve ileal morphology and decrease content of proinflammatory cytokines, which were associated with alterations in ileal microbiota induced by xylanase supplementation. The positive effects of xylanase on the intestinal health were more pronounced in young broilers, indicating an adaptive effect was exhibited as broilers aged.

\section{DATA AVAILABILITY STATEMENT}

The datasets presented in this study can be found in online repositories. The names of the repository/repositories and accession number(s) can be found at: https://www.ncbi.nlm. nih.gov/, PRJNA725811. 


\section{ETHICS STATEMENT}

The animal study was reviewed and approved by The Laboratory Animal Welfare and Animal Experimental Ethical Inspection Committee of China Agricultural University (Beijing, China).

\section{AUTHOR CONTRIBUTIONS}

JW and XP conceived and designed the experiments. JW, SL, and JM performed the animal experiments. JW analyzed

\section{REFERENCES}

Apajalahti, J., Kettunen, A., and Graham, H. (2004). Characteristics of the gastrointestinal microbial communities, with special reference to the chicken. Worlds Poult. Sci. J. 60, 223-232. doi: 10.1079/WPS20040017

Arana, A. T., Valle, J., Solano, C., Arrizubieta, M. J., Cucarella, C., Lamata, M. E., et al. (2001). The Enterococcal surface protein, Esp, is involved in Enterococcus faecalis biofilm formation. Appl. Environ. Microbiol. 67, 4538-4545. doi: 10.1128/AEM.67.10.4538-4545.2001

Ball, M. E. E., Owens, B., and McCracken, K. J. (2013). Chemical and physical predictors of the nutritive value of wheat in broiler diets. Asian Aust. J. Anim. Sci. 26, 97-107. doi: 10.5713/ajas.2012.12178

Bar-Shira, E., Sklan, D., and Friedman, A. (2003). Establishment of immune competence in the avian GALT during the immediate post-hatch period. Dev. Comp. Immunol. 27, 147-157. doi: 10.1016/S0145-305X(02)00076-9

Bautil, A., Verspreet, J., Buyse, J., Goos, P., Bedford, M. R., and Courtin, C. M. (2019). Age-related arabinoxylan hydrolysis and fermentation in the gastrointestinal tract of broilers fed wheat-based diets. Poult. Sci. 98, 4606-4621. doi: $10.3382 / \mathrm{ps} / \mathrm{pez} 159$

Bedford, M. R. (2018). The evolution and application of enzymes in the animal feed industry: the role of data interpretation. Br. Poult. Sci. 59, 486-493. doi: $10.1080 / 00071668.2018 .1484074$

Bedford, M. R., and Schulze, H. (1998). Exogenous enzymes for pigs and poultry. Nutr. Res. Rev. 11, 91-114. doi: 10.1079/NRR19980007

Camilleri, M., Madsen, K., Spiller, R., Van Meerveld, B. G., and Verne, G. N. (2012). Intestinal barrier function in health and gastrointestinal disease. Neurogastroenterol. Motil. 24, 503-512. doi: 10.1111/j.1365-2982.2012.01921.x

Cardoso, V., Fernandes, E. A., Santos, H., Macas, B., Lordelo, M. M., Telo, D. G. L., et al. (2018). Variation in levels of non-starch polysaccharides and endogenous endo-1,4-beta-xylanases affects the nutritive value of wheat for poultry. Br. Poult. Sci. 59, 218-226. doi: 10.1080/00071668.2018.1423674

Chadfield, M. S., Christensen, J. P., Juhl-Hansen, J., Christensen, H., and Bisgaard, M. (2005). Characterization of Enterococcus hirae outbreaks in broiler flocks demonstrating increased mortality because of septicemia and endocarditis and/or altered production parameters. Avain. Dis. 49, 16-23. doi: $10.1637 / 7205-050604$

Chen, L. P., Li, H., Li, J., Chen, Y., and Yang, Y. (2019). Lactobacillus rhamnosus GG treatment improves intestinal permeability and modulates microbiota dysbiosis in an experimental model of sepsis. Int. J. Mol. Med. 43, 1139-1148. doi: $10.3892 /$ ijmm.2019.4050

Chen, H., Zhang, S., and Kim, S. W. (2020). Effects of supplemental xylanase on health of the small intestine in nursery pigs fed diets with corn distillers' dried grains with solubles. J. Anim. Sci. 98:skaa185. doi: 10.1093/jas/skaa185

Choct, M., Hughes, R. J., and Bedford, M. R. (1999). Effects of a xylanase on individual bird variation, starch digestion throughout the intestine, and ileal and caecal volatile fatty acid production in chickens fed wheat. Br. Poult. Sci. 40, 419-422. doi: 10.1080/00071669987548

Choct, M., Hughes, R. J., Wang, J., Bedford, M. R., Morgan, A. J., and Annison, G. (1996). Increased small intestinal fermentation is partly responsible for the anti-nutritive activity of non-starch polysaccharides in chickens. Br. Poult. Sci. 37, 609-621. doi: 10.1080/00071669608417891

Dai, D., Qiu, K., Zhang, H., Wu, S., Han, Y., Wu, Y., et al. (2021). Organic acids as alternatives for antibiotic growth promoters alter the intestinal the data and wrote the manuscript. XP supervised and provided continuous guidance for the experiments. All authors have discussed the results and reviewed the manuscript.

\section{FUNDING}

Our study was supported by the National Natural Science Foundation of China (31772612) and the Beijing Municipal Natural Science Foundation (6202019).

structure and microbiota and improve the growth performance in broilers. Front. Microbiol. 11:618144. doi: 10.3389/fmicb.2020.618144

Dai, D., Wu, S., Zhang, H., Qi, G., and Wang, J. (2020). Dynamic alterations in early intestinal development, microbiota and metabolome induced by in ovo feeding of L-arginine in a layer chick model. J. Anim. Sci. Biotechnol. 11:19. doi: 10.1186/s40104-020-0427-5

Danicke, S., Vahjen, W., Simon, O., and Jeroch, H. (1999). Effects of dietary fat type and xylanase supplementation to rye-based broiler diets on selected bacterial groups adhering to the intestinal epithelium. On transit time of feed, and on nutrient digestibility. Poult. Sci. 78, 1292-1299. doi: 10.1093/ ps/78.9.1292

DiCenzo, G. C., Mengoni, A., and Perrin, E. (2019). Chromids aid genome expansion and functional diversification in the family Burkholderiaceae. Mol. Biol. Evol. 36, 562-574. doi: 10.1093/molbev/msy248

Duarte, M. E., Zhou, F. X., Dutra, W. M., and Kim, S. W. (2019). Dietary supplementation of xylanase and protease on growth performance, digesta viscosity, nutrient digestibility, immune and oxidative stress status, and gut health of newly weaned pigs. Anim. Nutr. 5, 351-358. doi: 10.1016/j. aninu.2019.04.005

Engberg, R. M., Hedemann, M. S., Steenfeldt, S., and Jensen, B. B. (2004). Influence of whole wheat and xylanase on broiler performance and microbial composition and activity in the digestive tract. Poult. Sci. 83, 925-938. doi: $10.1093 / \mathrm{ps} / 83.6 .925$

Fanning, A. S., Jameson, B. J., Jesaitis, L. A., and Anderson, J. M. (1998). The tight junction protein ZO-1 establishes a link between the transmembrane protein occludin and the actin cytoskeleton. J. Biol. Chem. 273, 29745-29753. doi: $10.1074 /$ jbc.273.45.29745

Funke, G., Haase, G., Schnitzler, N., Schrage, N., and Reinert, R. R. (1997) Endophthalmitis due to Microbacterium species:case report and review of Microbacterium infections. Clin. Infect. Dis. 24, 713-716. doi: 10.1093/ clind/24.4.713

Gao, F., Jiang, Y., Zhou, G. H., and Han, Z. K. (2007). The effects of xylanase supplementation on growth, digestion, circulating hormone and metabolite levels, immunity and gut microflora in cockerels fed on wheat-based diets. Br. Poult. Sci. 48, 480-488. doi: 10.1080/00071660701477320

Gao, P., Ma, C., Sun, Z., Wang, L., Huang, S., Su, X., et al. (2017). Feedadditive probiotics accelerate yet antibiotics delay intestinal microbiota maturation in broiler chicken. Microbiome 5:91. doi: 10.1186/ s40168-017-0315-1

Gawe, S., Wardas, M., Niedworok, E., and Wardas, P. (2004). Malondialdehyde (MDA) as a lipid peroxidation marker. Wiad. Lek. 57, 453-455.

González-Ortiz, G., Olukosi, O., and Bedford, M. R. (2016). Evaluation of the effect of different wheats and xylanase supplementation on performance, nutrient and energy utilisation in broiler chicks. Anim. Nutr. 2, 173-179. doi: 10.1016/j.aninu.2016.06.005

Hotterbeekx, A., Xavier, B. B., Bielen, K., Lammens, C., Moons, P., Schepens, T. et al. (2016). The endotracheal tube microbiome associated with Pseudomonas aeruginosa or Staphylococcus epidermidis. Sci. Rep. 6:36507. doi: 10.1038/ srep36507

Hübener, K., Vahjen, W., and Simon, O. (2002). Bacterial responses to different dietary cereal types and xylanase supplementation in the intestine of broiler chicken. Arch. Anim. Nutr. 56, 167-187. doi: 10.1080/00039420214191

Jensen, D. M., Hendricks, K. V., Mason, A. T., and Tessem, J. S. (2020). Good cop, bad cop: the opposing effects of macrophage activation state on 
maintaining or damaging functional $\beta$-cell mass. Metabolites 10:485. doi: $10.3390 /$ metabo10120485

Józefiak, D., Rutkowski, A., Jensen, B. B., and Engberg, R. M. (2007). Effects of dietary inclusion of triticale, rye and wheat and xylanase supplementation on growth performance of broiler chickens and fermentation in the gastrointestinal tract. Anim. Feed Sci. Technol. 132, 79-93. doi: 10.1016/j. anifeedsci.2006.03.011

Lee, S. H., Hosseindoust, A., Laxman Ingale, S., Rathi, P. C., Yoon, S. Y., Choi, J. W., et al. (2020). Thermostable xylanase derived from Trichoderma citrinoviride increases growth performance and non-starch polysaccharide degradation in broiler chickens. Br. Poult. Sci. 61, 57-62. doi: 10.1080/00071668.2019.1673316

Li, C., Wang, J., Zhang, H., Wu, S., Hui, Q., Yang, C., et al. (2019). Intestinal morphologic and microbiota responses to dietary Bacillus spp. in a broiler chicken model. Front. Physiol. 9:1968. doi: 10.3389/fphys.2018.01968

Lim, G. E., Huang, G. J., Flora, N., LeRoith, D., Rhodes, C. J., and Brubaker, P. (2009). Insulin regulates glucagon-like peptide-1 secretion from the enteroendocrine L cell. Endocrinology 150, 580-591. doi: 10.1210/en.2008-0726

Liu, D., Guo, S., and Guo, Y. (2012). Xylanase supplementation to a wheatbased diet alleviated the intestinal mucosal barrier impairment of broiler chickens challenged by Clostridium perfringens. Avian Pathol. 41, 291-298. doi: 10.1080/03079457.2012.684089

Liu, P., Zhao, J., Guo, P., Lu, W., Geng, Z., Levesque, C. L., et al. (2017). Dietary corn bran fermented by Bacillus subtilis MA139 decreased gut cellulolytic bacteria and microbiota diversity in finishing pigs. Front. Cell. Infect. Microbiol. 7:526. doi: 10.3389/fcimb.2017.00526

Livak, K. J., and Schmittgen, T. D. (2001). Analysis of relative gene expression data using real-time quantitative PCR and the $2^{-\Delta \Delta C T}$ method. Methods 25, 402-408. doi: 10.1006/meth.2001.1262

Lji, P. A., Saki, A., and Tivey, D. R. (2001). Body and intestinal growth of broiler chicks on a commercial starter diet. 1. Intestinal weight and mucosal development. Br. Poult. Sci. 42, 505-513. doi: 10.1080/00071 660120073151

Luo, D., Yang, F., Yang, X., Yao, J., Shi, B., and Zhou, Z. (2009). Effects of xylanase on performance, blood parameters, intestinal morphology, microflora and digestive enzyme activities of broilers fed wheat-based diets. Asian. Aust. J. Anim. Sci. 22, 1288-1295. doi: 10.5713/ajas.2009.90052

Mahfuz, S., He, T., Liu, S., Wu, D., Long, S., and Piao, X. (2019). Dietary inclusion of mushroom (Flammulina velutipes) stem waste on growth performance, antibody response, immune status, and serum cholesterol in broiler chickens. Animals 9:692. doi: 10.3390/ani9090692

Mantell, M. P., Ziegler, T. R., Adamson, W. T., Roth, J. A., Zhang, W., Frankel, W., et al. (1995). Resection-induced colonic adaptation is augmented by IGF-I and associated with upregulation of colonic IGF-I mRNA. Am. J. Phys. 269, G974-G980. doi: 10.1152/ajpgi.1995.269.6.G974

Masey, O., Neill, H. V., Smith, J. A., and Bedford, M. R. (2014). Multicarbohydrase enzymes for non-ruminants. Asian. Aust. J. Anim. Sci. 27, 290-301. doi: 10.5713/ajas.2013.13261

McCafferty, K. W., Bedford, M. R., Kerr, B. J., and Dozier, W. A. (2019a). Effects of age and supplemental xylanase in corn- and wheat-based diets on cecal volatile fatty acid concentrations of broilers. Poult. Sci. 98, 47874800. doi: 10.3382/ps/pez194

McCafferty, K. W., Bedford, M. R., Kerr, B. J., and Dozier, W. A. (2019b). Effects of cereal grain source and supplemental xylanase concentrations on broiler growth performance and cecal volatile fatty acid concentrations from 1 to $40 \mathrm{~d}$ of age. Poult. Sci. 98, 2866-2879. doi: 10.3382/ps/pez032

National Research Council (1994). Nutrient Requirements of Poultry. 9th Edn. Washington, DC: The National Academies Press, 176.

Neveling, D. P., van Emmenes, L., Ahire, J. J., Pieterse, E., Smith, C., and Dicks, L. M. T. (2017). Safety assessment of antibiotic and probiotic feed additives for Gallus gallus domesticus. Sci. Rep. 7:12767. doi: 10.1038/ s41598-017-12866-7

Petry, A. L., Huntley, N. F., Bedford, M. R., and Patience, J. F. (2020). Xylanase increased the energetic contribution of fiber and improved the oxidative status, gut barrier integrity, and growth performance of growing pigs fed insoluble corn-based fiber. J. Anim. Sci. 98:skaa233. doi: 10.1093/jas/skaa233

Petry, A. L., Patience, J. F., Nichole, F. H., Koester, L. R., Bedford, M. R., and Schmitz-Esser, S. (2021). Xylanase supplementation modulates the microbiota of the large intestine of pigs fed corn-based fiber by means of a stimbiotic mechanism of action. Front. Microbiol. 12:619970. doi: 10.3389/ fmicb.2021.619970

Ramanan, P., Barreto, J. N., Osmon, D. R., and Tosh, P. K. (2014). Rothia bacteremia: a 10-year experience at Mayo Clinic, Rochester, Minnesota. J. Clin. Microbiol. 52, 3184-3189. doi: 10.1128/JCM.01270-14

Raza, A., Bashir, S., and Tabassum, R. (2019). An update on carbohydrases: growth performance and intestinal health of poultry. Heliyon 5:e1437. doi: 10.1016/j.heliyon.2019.e01437

Rizzatti, G., Lopetuso, L. R., Gibiino, G., Binda, C., and Gasbarrini, A. (2017). Proteobacteria: a common factor in human diseases. Biomed. Res. Int 2017:9351507. doi: 10.1155/2017/9351507

Rosicka-Kaczmarek, J., Komisarczyk, A., Nebesny, E., and Makowski, B. (2016). The influence of arabinoxylans on the quality of grain industry products. Eur. Food Res. Technol. 242, 295-303. doi: 10.1007/s00217-015-2549-0

Rychlik, I. (2020). Composition and function of chicken gut microbiota. Animals 10:103. doi: 10.3390/ani10010103

Schokker, D., Jansman, A. J. M., Veninga, G., de Bruin, N., Vastenhouw, S. A., de Bree, F. M., et al. (2017). Perturbation of microbiota in one-day old broiler chickens with antibiotic for 24 hours negatively affects intestinal immune development. BMC Genomics 18:241. doi: 10.1186/s12864-017-3625-6

Smeets, N., Nuyens, F., Van Campenhout, L., Delezie, E., and Niewold, T. A. (2018). Interactions between the concentration of non-starch polysaccharides in wheat and the addition of an enzyme mixture in a broiler digestibility and performance trial. Poult. Sci. 97, 2064-2070. doi: 10.3382/ps/pey038

Sudhir, Y., and Jha, R. (2019). Strategies to modulate the intestinal microbiota and their effects on nutrient utilization, performance, and health of poultry. J. Anim. Sci. Biotechnol. 10:2. doi: 10.1186/s40104-018-0310-9

Tiwari, U. P., Chen, H., Kim, S. W., and Jha, R. (2018). Supplemental effect of xylanase and mannanase on nutrient digestibility and gut health of nursery pigs studied using both in vivo and in vitro models. Anim. Feed Sci. Technol. 245, 77-90. doi: 10.1016/j.anifeedsci.2018.07.002

Umare, V., Pradha, V., Nadka, M., Rajadhyaksha, A., Patwardhan, M., Ghosh, K. K., et al. (2014). Effect of proinflammatory cytokines (IL-6, TNF- $\alpha$, and IL-1 $\beta$ ) on clinical manifestations in Indian SLE patients. Mediat. Inflamm. 2014:385297. doi: $10.1155 / 2014 / 385297$

Wagner, B. D., Grunwald, G. K., Zerbe, G. O., Mikulich-Gilbertson, S. K., Robertson, C. E., and Zemanick, E. T. (2018). On the use of diversity measures in longitudinal sequencing studies of microbial communities. Front. Microbiol. 9:1037. doi: 10.3389/fmicb.2018.01037

Wang, Y., Heng, C., Zhou, X., Cao, G., Jiang, L., Wang, J., et al. (2021). Supplemental Bacillus subtilis DSM 29784 and enzymes, alone or in combination, as alternatives for antibiotics to improve growth performance, digestive enzyme activity, anti-oxidative status, immune response and the intestinal barrier of broiler chickens. Brit. J. Nutr. 125, 494-507. doi: 10.1017/ S0007114520002755

Wang, J., Liu, Y., Yang, Y., Bao, C., and Cao, Y. (2020). High-level expression of an acidic thermostable xylanase in Pichia pastoris and its application in weaned piglets. J. Anim. Sci. 98:skz364. doi: 10.1093/jas/skz364

Wang, H., Ni, X., Qing, X., Zeng, D., Luo, M., Liu, L., et al. (2017). Live probiotic Lactobacillus johnsonii BS15 promotes growth performance and lowers fat deposition by improving lipid metabolism, intestinal development, and gut microflora in broilers. Front. Microbiol. 8:1073. doi: 10.3389/ fmicb.2017.01073

Wu, D., Lewis, E. D., Pae, M., and Meydani, S. N. (2018). Nutritional modulation of immune function: analysis of evidence, mechanisms, and clinical relevance. Front. Immunol. 9:3160. doi: 10.3389/fimmu.2018.03160

Wu, Y. B., Ravindran, V., Thomas, D. G., Birtles, M. J., and Hendriks, W. H. (2004a). Influence of method of whole wheat inclusion and xylanase supplementation on the performance, apparent metabolisable energy, digestive tract measurements and gut morphology of broilers. Br. Poult. Sci. 45, 385-394. doi: 10.1080/00071660410001730888

Wu, Y. B., Ravindran, V., Thomas, D. G., Birtles, M. J., and Hendriks, W. H. (2004b). Influence of phytase and xylanase, individually or in combination, on performance, apparent metabolisable energy, digestive tract measurements and gut morphology in broilers fed wheat-based diets containing adequate level of phosphorus. Br. Poult. Sci. 45, 76-84. doi: 10.1080/00071660 410001668897 
Zhang, S., Wang, C., Sun, Y., Wang, G., Chen, H., Li, D., et al. (2018). Xylanase and fermented polysaccharide of Hericium caputmedusae reduce pathogenic infection of broilers by improving antioxidant and antiinflammatory properties. Oxidative Med. Cell. Longev. 2018:4296985. doi: $10.1155 / 2018 / 4296985$

Zhang, L., Xu, J., Lei, L., Jiang, Y., Gao, F., and Zhou, G. H. (2014). Effects of xylanase supplementation on growth performance, nutrient digestibility and non-starch polysaccharide degradation in different sections of the gastrointestinal tract of broilers fed wheat-based diets. Asian Australas. J. Anim. Sci. 27, 855-861. doi: 10.5713/ajas.2014.14006
Conflict of Interest: The authors declare that the research was conducted in the absence of any commercial or financial relationships that could be construed as a potential conflict of interest.

Copyright $\odot 2021$ Wang, Liu, Ma and Piao. This is an open-access article distributed under the terms of the Creative Commons Attribution License (CC BY). The use, distribution or reproduction in other forums is permitted, provided the original author(s) and the copyright owner(s) are credited and that the original publication in this journal is cited, in accordance with accepted academic practice. No use, distribution or reproduction is permitted which does not comply with these terms. 